\title{
An admittance shaping controller for exoskeleton assistance of the lower extremities
}

\author{
Gabriel Aguirre-Ollinger • Umashankar Nagarajan • Ambarish Goswami •
}

Received: date / Accepted: date

\begin{abstract}
We present a method for lower-limb exoskeleton control that defines assistance as a desired dynamic response for the human leg. Wearing the exoskeleton can be seen as replacing the leg's natural admittance with the equivalent admittance of the coupled system. The control goal is to make the leg obey an admittance model defined by target values of natural frequency, peak magnitude and zero-frequency response. No estimation of muscle torques or motion intent is necessary. Instead, the controller scales up the coupled system's sensitivity transfer function by means of a compensator employing positive feedback. This approach increases the leg's mobility and makes the exoskeleton an active device capable of performing net positive work on the limb. Although positive feedback is usually considered destabilizing, here performance and robust stability are successfully achieved through a constrained optimization that maximizes the system's gain margins while ensuring the desired location of its dominant poles.
\end{abstract}

Keywords Exoskeleton - Assistive robotics · Interaction control $\cdot$ Admittance control

\section{Notation}

Our control method is formulated in terms of Laplace-domain transfer functions. Here we explain the notation employed:

- Transfer functions.

This research was supported by a grant from the Honda Research Institute, Mountain View, CA, USA.

G. Aguirre-Ollinger

Centre for Autonomous Systems, University of Technology, Sydney, Broadway 2007, Australia

E-mail: gabriel.aguirre-ollinger@uts.edu.au

U. Nagarajan · A. Goswami

Honda Research Institute, Mountain View, CA 94043, USA
- $Z_{-}(s)$ : mechanical impedance.

- $Y_{-}(s)$ : mechanical admittance.

- $X_{-}(s)$ : integral of the mechanical admittance $\left(X_{-}(s)=\right.$ $\left.Y_{-}(s) / s\right)$.

- $S_{-}(s)$ : sensitivity transfer function. This is a closedloop transfer function that evaluates to 1 at all frequencies when the feedback gain is zero.

- $T_{-}(s)$ : complementary sensitivity transfer function, given by $T_{-}(s)=1-S_{-}(s)$.

- $L_{-}(s)$ : loop transfer function for root-locus analysis.

- $N_{-}(s)$ : numerator of a rational transfer function.

- $D_{-}(s)$ : denominator of a rational transfer function.

- $W_{-}(s)$ : loop transfer function for robustness analysis (sec.4).

- Subscripts are used to indicate which subsystems are present in a particular transfer function.

- $h$ : human leg.

- $e$ : exoskeleton mechanism, consisting of the actuator and arm.

- $c$ : compliant coupling between the human leg and the exoskeleton mechanism, modeled as a spring and damper.

- $f$ : feedback compensator for the exoskeleton.

\section{Introduction}

Exoskeletons are wearable mechanical devices that possess a kinematic configuration similar to that of the human body and have the ability to follow the movements of the user's extremities. Powered exoskeletons are usually designed to produce contact forces to assist the user in performing a motor task. In recent years, a large number of lower-limb exoskeleton systems and their associated control methods have been developed, either as research tools for the study of human gait (Ferris et al, 2007; Emken et al, 2006), or as reha- 
bilitation tools for patients with stroke and other locomotor disorders (Dollar and Herr, 2008b). In a parallel development, a number of lightweight, autonomous exoskeletons have been designed with the aim of assisting impaired or aged users in daily-living situations (Ekso Bionics ${ }^{\mathrm{TM}}, 2013$; American Honda Motor Co.. Inc., 2009). This research aims to develop a general-purpose exoskeleton control that minimizes the need for estimating the user's intended motion. Our primary target application is autonomous exoskeletons for daily living.

\subsection{A classification of exoskeleton-based assistive strategies}

Together with the physical exoskeletons, a wide variety of assistive strategies have been developed and tested with varying levels of success. Below we present a compact classification of strategies for aiding human locomotion, along with a few examples that are currently available in the literature.

1. Based on what aspect of the body's movement is supported by the assistive forces or torques.

(a) Propulsion of the body's center of mass, especially during the stance phase of walking (Kazerooni et al, 2005; Blaya and Herr, 2004).

(b) Propulsion of the unconstrained leg, for example during the swing phase of walking (Veneman et al, 2005; Banala et al, 2009).

(c) Gravitational support of the extremities (Banala et al, 2006).

2. By the intended effect on the dynamics or physiology of human movement.

(a) Reducing the muscle activation required for walking at a given speed (Kawamoto et al.2003; Gordon et al, 2013).

(b) Increasing the comfortable walking speed for a given level of muscle effort (Norris et al, 2007). This could be attained either through an increase in mean stride length (Sawicki and Ferris, 2009) or mean stepping frequency (Lee and Sankai, 2003).

(c) Reducing the metabolic cost of walking (Sawicki and Ferris, 2008; Mooney et al, 2014).

(d) Correcting anomalies of the gait trajectory (Banala et al, 2009; Van Asseldonk et al, 2007).

(e) Balance recovery and dynamic stability during walking (European Commission (CORDIS), 2013).

It should be noted that the assistive strategies in the second category occur on different time scales. The effects sought can range from immediate, as in the case of balance recovery and dynamic stability, to long-term as in the case of gait anomaly correction, which normally only becomes apparent over the course of several training sessions.

\subsection{Current exoskeleton control methods}

In order to realize the chosen assistive strategy it is necessary to design an appropriate exoskeleton control. The prevalent view is that control of the walking task must be shared by the user and the exoskeleton, with the device allowing for the user's intention and voluntary efforts (Hogan et al, 2006; Vallery et al, 2009b; Bernhardt et al, 2005). Strategies for shared control include timing the exoskeleton's response to the phases of the gait cycle (Blaya and Herr. 2004; Kawamoto and Sankai, 2005; Malcolm et al, 2013), leading the patient towards a clinically correct trajectory via soft constraints (Banala et al, 2009) and modifying the dynamic response of the lower limbs by means of active admittance (AguirreOllinger et al, 2011) or generalized elasticities (Vallery et al, 2009a). Also, the view of human gait as a stable limit cycle has led to the emergence of oscillator-based exoskeleton control. Relevant methods include adding energy at resonance via a phase oscillator (Sugar et al, 2015), and synchronizing exoskeleton torques to the user's lower-limb trajectory (Ronsse et al, 2011) or to muscle torques (AguirreOllinger, 2015, 2013) using adaptive frequency oscillators (AFOs).

One of the simplest strategies for exoskeleton control is to exploit the uniformity of the human gait cycle when walking at a constant speed. This approach has been employed on a number of treadmill-based exoskeletons. For example, the pneumatically powered exoskeleton described in Lewis and Ferris (2011) uses a footswitch-generated signal to power the device's actuators for a predetermined portion of the gait cycle. The hip exoskeleton reported in Lenzi et al (2013) computes the current stride percent by means of an AFO, and uses it to deliver an assistive torque proportional to the nominal hip torque profile of human gait. Although these systems have proven effective, they are limited by definition to assisting uniform-speed gait. Our research is motivated by the desire to have a more versatile control, less dependent on gait uniformity, and capable of assisting other lowerlimb movements like gait initiation and reactive stepping. Our overarching goal is a control that provides assistance independently of the specific motion attempted.

\subsection{Lower-limb assistance as a desired dynamic response}

Our approach to exoskeleton control defines assistance in terms of a desired dynamic response for the leg, specifically a desired mechanical admittance. If we model the leg dynamics as the transfer function of a linear time-invariant (LTI) system, its admittance is a single- or multiple-port transfer function relating the net muscle torque acting on each joint to the resulting angular velocities of the joints. When the exoskeleton is coupled to the leg, the admittance 
of the human leg gets replaced, in a sense, by the equivalent admittance of the coupled system.

The idea behind our method is to make this admittance modification work to the user's advantage. The resulting admittance of the assisted leg should facilitate the motion of the lower extremities, for example by reducing the muscle torque needed to accomplish a certain movement, or by enabling quicker point-to-point movements than the user can accomplish without assistance. The immediate advantage of this approach is that it does not require predicting the user's intended motion or attempting to track a prescribed motion trajectory.

\subsection{Contributions}

Our control method, which we will refer to as admittance shaping, is formulated in the language of linear control theory. The overall design objective is to make the equivalent admittance of the assisted leg meet certain specifications of frequency response. Once this desired admittance has been defined, the design problem consists of generating a port impedance on the exoskeleton (through state feedback) such that, when the exoskeleton is attached to the human limb, the coupled system exhibits the desired admittance characteristics. Thus our problem is properly classified as one of interaction controller design (Buerger and Hogan, 2007).

This paper presents a formulation of admittance shaping control for single-joint motion which employs linearized models of the exoskeleton and the human limb. The control is designed mainly to assist the leg during the swing phase of walking, where the leg's pendulum-like dynamics prevail. Accordingly, we have modeled the leg as a 1-DOF rotational pendulum, and investigated the way to modify the dynamic properties of said pendulum, mainly natural frequency and damping, by means of an exoskeleton. The link between this effect and the dynamics of bipedal walking is analyzed briefly in a simulation study.

This research is a generalization of exoskeleton controls we have previously developed around the idea of making the exoskeleton's admittance active. Said controls involved emulated inertia compensation (Aguirre-Ollinger et al, 2011, 2012) or negative damping (Aguirre-Ollinger et al, 2007). Although the notion of modifying the dynamics of the human limb is somehow implicit in methods like the "subject comfort" control of the HAL exoskeleton (Kawamoto and Sankai, 2005) and the generalized elasticities control proposed by Vallery (Vallery et al, 2009a), in those methods the exoskeleton's port impedance remains passive, and as such does not perform net work on the human limb; an additional layer of active control is required.

Our control renders the exoskeleton port impedance active by means of positive feedback of the exoskeleton's kine-

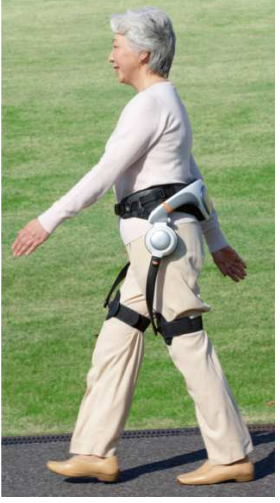

(a)

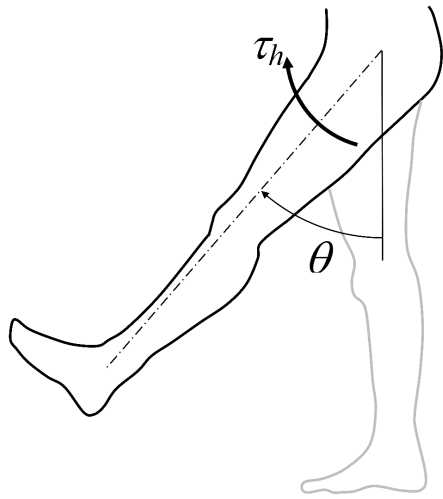

(b)
Fig. 1 (a) Stride Management Assist device: a powered autonomous exoskeleton for gait assistance (Honda Motor Co., Ltd.). (b) Extended human leg: sagittal plane view.

matic state. This approach has some similarity with the control of the BLEEX exoskeleton Kazerooni et al, 2005), in which positive feedback makes the device highly responsive to the user's movements. However, in that system the actual assistance comes in the form of gravitational support of an external load. By contrast, our interaction controller makes positive feedback the source of the assistive effect.

The design of our interaction controller solves two problems concurrently: the performance problem, i.e. producing the desired admittance, and the stabilization of the coupled system. As we shall show, for the exoskeleton's assistive control the dynamic response objectives embodied by the desired admittance tend to trade off against the stability margins of the coupled system. Equally important is the fact that our coupled system involves a considerable level of parameter uncertainty, especially when it comes to the dynamic parameters of the leg and the parameters of the coupling between the leg and the exoskeleton. Therefore the design needs to ensure a sufficient level of stability and performance robustness.

The present study covers the following aspects:

- Formulation of the assistive effect in terms of a target admittance (and the integral thereof) for the assisted leg (section 2).

- Design of the exoskeleton's assistive control proper (section 3). A central topic is the use of positive feedback control and how to ensure the stability of the coupled system.

- Robust stability analysis of the control (section 4).

- Simulation of assisted bipedal walk and initial tests of the exoskeleton control on human subjects (section 5). 

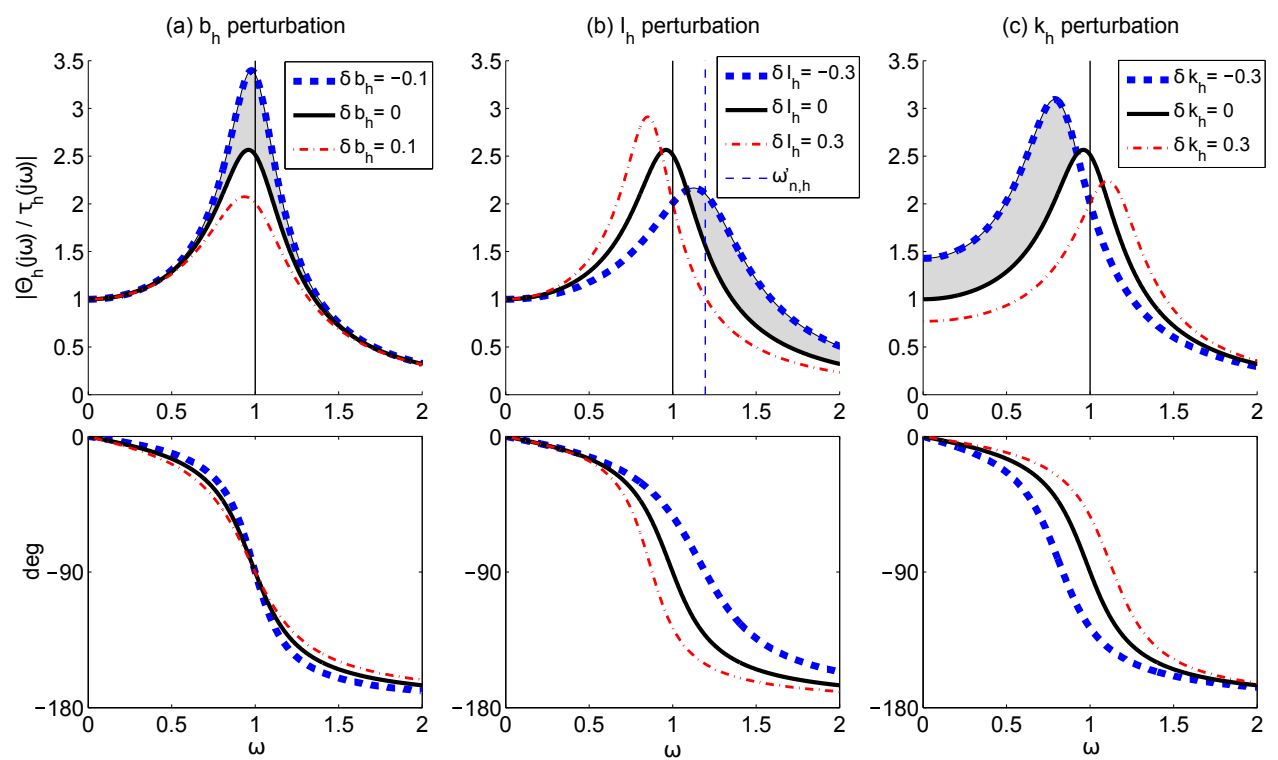

Fig. 2 Effects of impedance perturbations on the frequency response (magnitude ratio and phase) of the integral admittance of the human leg: (a) damping perturbations; (b) inertia perturbations; (c) stiffness perturbations. Effects of both negative and positive perturbations are shown. Gray areas highlight portions where a negative perturbation causes a reduction in magnitude ratio. For a given angle amplitude, these areas represent "effort reduction", i.e. a reduction in required muscle torque amplitude with respect to the unperturbed admittance.

\section{Admittance shaping: formulation of the assistive effect in terms of a target leg admittance}

In this section we present three basic forms of assistance modeled as perturbations of the human leg's inertia, damping and/or stiffness. Then we give a general-purpose definition of exoskeleton assistance formulated in terms of the limb's sensitivity transfer function. This transfer function provides a measure of how the leg's dynamic response is affected by the perturbations. The definition is formulated using the Bode sensitivity integral theorem (Middleton and Braslavsky, 2000). As we will show, this theorem provides a general avenue for the design of the assistive control, namely the use of positive feedback of the exoskeleton's kinematic state.

In order to develop our mathematical formulation of lowerlimb assistance, we shall use a specific exoskeleton system as a motivating example. The Stride Management Assist (SMA) device, shown in Figure 1(a) is an autonomous powered exoskeleton developed by Honda Motor Co., Ltd. (Japan). The system features two flat brushless motors concentric with the axis of the hip joints on the sagittal plane. The motors exert torque on the user's legs through a pair of rigid, lightweight arms coupled to the thighs. This configuration makes the SMA device especially effective in assisting the swing phase of the walking cycle as well as other leg movements not involving ground contact.

In the human gait cycle, the swing phase takes advantage of pendulum dynamics of the leg (Kuo, 2002; Sugar et al, 2015). Therefore, for the present study we shall model the leg as a linear rotational pendulum. This model is an approx- imate representation of the extended leg swinging about the hip joint on the sagittal plane (Figure 1(b). The impedance of the leg at the hip joint, $Z_{h}(s)$, is the transfer function relating the net muscle torque acting on that joint, $\tau_{h}(s)$, to the resulting angular velocity of the leg $\Omega_{h}(s)$ :

$Z_{h}(s)=\frac{\tau_{h}(s)}{\Omega_{h}(s)}=I_{h} s+b_{h}+\frac{k_{h}}{s}$

where $I_{h}$ is the moment of inertia of the leg about the hip joint, and $b_{h}$ and $k_{h}$ are, respectively, the damping and stiffness coefficients of the joint. The coefficient $k_{h}$ includes both the stiffness the joint's structure and a linearization of the action of gravity on the leg. The following are typical values of the hip joint's dynamic parameters in adult subjects:

- Moment of inertia of the extended leg about the hip: 2.09 $\mathrm{kg} \cdot \mathrm{m}^{2}$ (Royer and Martin, 2005).

- Hip joint damping coefficient: $3.5 \mathrm{Nm} \mathrm{s} / \mathrm{rad}$ (Tafazzoli and Lamontagne, 1996).

- Natural pendulum frequency of the extended leg: 0.64 $\mathrm{Hz}$ (Ghista, 2008; Doke et al, 2005).

- Stiffness coefficient of the hip joint vertical stance during the swing phase of the gait cycle: $38 \mathrm{Nm} / \mathrm{rad}$ (Frigo et al, 1996).

In order to make our treatment general, all transfer functions in this paper are expressed in terms of dimensionless variables. A unity moment of inertia is equal to the moment of inertia of the leg about the hip joint; a unity angular frequency equals the natural undamped frequency of the leg. 
Based on the above published data we set the damping ratio of the hip joint to $\zeta_{h}=0.2$. This yields the following values for the coefficients in (11): $I_{h}=1, b_{h}=0.4$ and $k_{h}=1$.

\subsection{Shaping the admittance via impedance perturbations}

We model the ideal effect of assisting the human limb as applying an additive perturbation $\delta Z_{h}$ to the limb's natural impedance $Z_{h}$; the perturbed impedance is simply

$$
\widetilde{Z}_{h}=Z_{h}+\delta Z_{h}
$$

An equivalent expression can be given in terms of the leg's admittance, $Y_{h}(s)=Z_{h}(s)^{-1}$. The perturbed admittance, $\widetilde{Y}_{h}(s)$, represents a negative feedback system formed by $Y_{h}$ and $\delta Z_{h}$ :

$\widetilde{Y}_{h}=\frac{1}{Z_{h}+\delta Z_{h}}=\frac{Y_{h}}{1+Y_{h} \delta Z_{h}}$

Our task is now to determine what makes $\delta Z_{h}$ assistive, i.e. what kind of perturbation makes $\widetilde{Y}_{h}$ an improvement over the leg's normal admittance $Y_{h}$. We note that each of the parameters $I_{h}, b_{h}$ and $k_{h}$ contributes to the magnitude of the leg's impedance (10; therefore we start by studying the effects of compensating each of the leg's dynamic properties. Accordingly we define the following perturbation types:

$$
\begin{aligned}
& \delta Z_{h}=\delta b_{h} \quad \text { (damping perturbation) } \\
& \delta Z_{h}=\delta I_{h} s \quad \text { (inertia perturbation) } \\
& \delta Z_{h}=\delta k_{h} / s \quad \text { (stiffness perturbation) }
\end{aligned}
$$

Compensation means that either $\delta b_{h}$ or $\delta I_{h}$ or $\delta k_{h}$ has a negative value. We now analyze the effects the individual perturbations on the frequency response of the integral admittance $\widetilde{Y}_{h}(s) / s$, which relates the net muscle torque to the angular position of the leg. We use this rather than the admittance in order to consider possible effects on the "DC gain" (zero-frequency response) as well.

Figure 2 shows the effects of each individual perturbation on the leg's integral admittance. Although our primary interest is compensation, we have also plotted the effects of positive perturbations for comparison. Examination of the plots reveals several properties of the perturbed frequency responses that can be considered as assistance:

- Damping compensation increases the peak magnitude of the integral admittance. In consequence, for given angular trajectories near the natural frequency $(\omega=1)$, the amplitude of the required muscle torque is reduced with respect to the unperturbed case. We refer to this effect as "effort reduction".

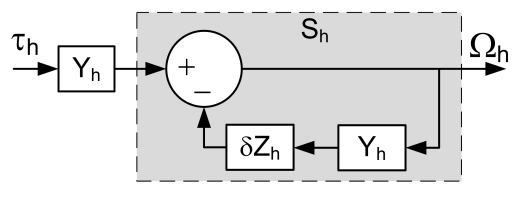

(a)

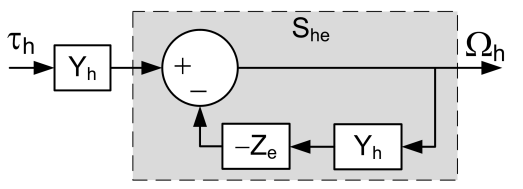

(b)

Fig. 3 (a) Block diagram representing the dynamics of the human leg in the presence of an impedance perturbation $\delta Z_{h}$. The perturbed admittance of the leg is equal to the nominal admittance $Y_{h}$ in series with a closed-loop system representing the sensitivity $S_{h}$. (b) Equivalent system formed by the human limb coupled to the exoskeleton. The perturbation $\delta Z_{h}$ is replaced by the exoskeleton transfer function $Z_{e}$. Per the Bode sensitivity integral theorem, to make the coupled system's sensitivity larger than the unperturbed case, positive feedback must be used.

- Inertia compensation causes an increase in the natural frequency of the leg with no change in DC gain. In consequence, given a desired amplitude of angular motion, the minimum muscle torque amplitude occurs now at a higher frequency. We hypothesize that the shift in natural frequency has a potential beneficial effects on the gait cycle. It may enable the user to walk at higher stepping frequencies without a significant increment in muscle activation (Doke and Kuo, 2007). A higher natural frequency also implies a quicker transient response, which may enable the user to take quicker reactive steps when trying to avoid a fall.

- Stiffness compensation produces effort reduction at frequencies below the natural frequency.

Each case has also its drawbacks. For example, the effect of damping compensation vanishes as motion frequency departs from the natural frequency. Inertia compensation causes effort increase at frequencies immediately below the natural frequency. However, by applying the principle of superposition, it is possible to devise a perturbation transfer function that combines the beneficial aspects of each individual perturbation. In other words, the resulting integral admittance can simultaneously feature increases in natural frequency, magnitude peak and DC gain with respect to the unassisted one.

As for perturbations involving positive values of $\delta b_{h}$, $\delta I_{h}$ and/or $\delta k_{h}$, we refer to these as resistive to indicate that they mainly tend to reduce the leg's mobility. 
(a) $b_{h}$ perturbation

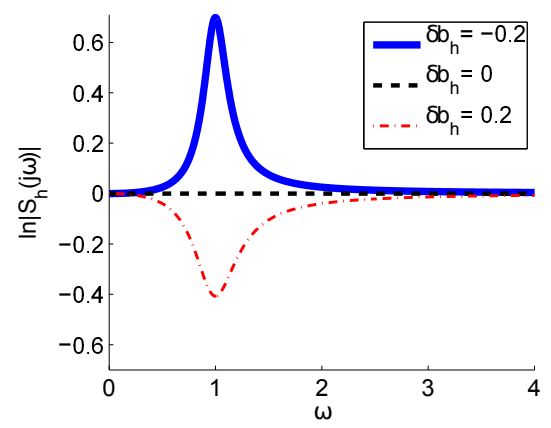

(b) $I_{h}$ perturbation

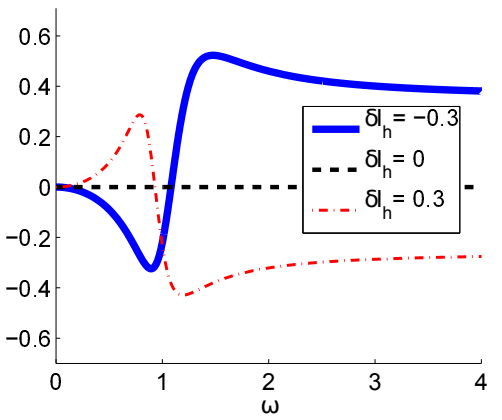

(c) $k_{h}$ perturbation

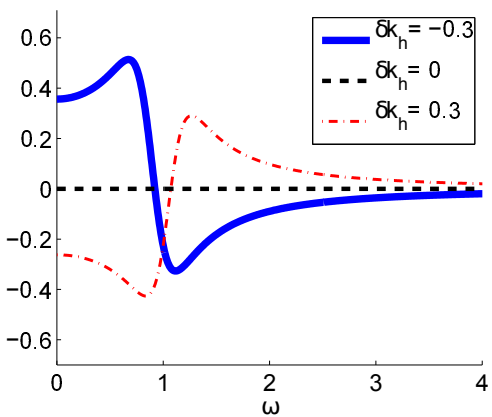

Fig. 4 Sensitivity plots for impedance perturbations $\left(\ln \left|S_{h}(j \omega)\right|\right)$. (a) Damping perturbation; (b) inertia perturbation; (c) stiffness perturbation.

2.2 Effect of impedance perturbations: the Bode sensitivity integral

The central problem covered in this study is how to design an exoskeleton controller capable of generating a virtual leg admittance with arbitrary properties of natural frequency, magnitude peak and DC gain. Our approach is to make the exoskeleton emulate the negative variations of $\delta b_{h}$, $\delta I_{h}$ and/or $\delta k_{h}$ described above. We now derive a general principle for the design of exoskeleton control: namely, when the leg is coupled to the exoskeleton, the leg's sensitivity to muscle torques should increase. We define the sensitivity transfer function $S_{h}(s)$ of the leg as

$S_{h}(s)=\frac{1}{1+Y_{h}(s) \delta Z_{h}(s)}=\frac{1+\frac{1}{I_{h}}\left(\frac{b_{h}}{s}+\frac{k_{h}}{s^{2}}\right)}{1+\frac{1}{I_{h}}\left(\frac{b_{h}}{s}+\frac{k_{h}}{s^{2}}+\frac{\delta Z_{h}}{s}\right)}$

If we consider muscle torque as the system's input and angular velocity as the output, $S_{h}$ provides a measure of how the system's input/output relationship is influenced by a perturbation $\delta Z_{h}$ to its dynamic parameters. In the absence of perturbations $S_{h}(j \omega)$ evaluates to 1 at all frequencies. Given an impedance perturbation $\delta Z_{h}, S_{h}(j \omega)$ can be seen as a weighting function that describes how the applied perturbation changes the shape of the leg's frequency response; the perturbed admittance is simply

$\tilde{Y}_{h}=S_{h} Y_{h}$

The perturbed admittance is shown in block diagram form in Figure 3(a). Here we restrict our analysis to perturbations whose effect vanishes at high frequencies, i.e. $\left|S_{h}\right| \rightarrow 1$ as $\omega \rightarrow \infty$. From (5) we see that this is the case for all but the inertia perturbation (4). However, the vanishing condition can easily be enforced by redefining the inertia perturbation as

$\delta Z_{h}=\delta I_{h} \frac{\omega_{o} s}{s+\omega_{o}}$
Choosing $\omega_{o} \gg 1$ (i.e. making it larger than the natural frequency of the leg) ensures that the perturbation maintains its desired behavior in the typical frequency range of leg motion.

A property of sensitivity transfer functions, known as the Bode sensitivity integral, will allow us to derive a general principle for the design of our exoskeleton control. The Bode sensitivity integral theorem (Middleton and Braslavsky, 2000) is stated as follows:

Let $L(s)$ be a proper, rational transfer function of relative degree $N_{r}$. (The relative degree of a transfer function is the difference between the order of the denominator and the order of the numerator.) Define the closed-loop sensitivity function $S(s)=(1+L(s))^{-1}$ and assume that neither $L(s)$ nor $S(s)$ have poles or zeros in the closed right half plane. Then,

$\int_{0}^{\infty} \ln |S(j \omega)| \mathrm{d} \omega= \begin{cases}0 & \text { if } N_{r}>1 \\ -\frac{\pi}{2} \lim _{s \rightarrow \infty} s L(s) & \text { if } N_{r}=1\end{cases}$

We now use the theorem to analyze the leg's sensitivity to perturbations by defining the loop transfer function $L_{h}(s)=Y_{h}(s) \delta Z_{h}(s)$. Evaluating the Bode sensitivity integral for the perturbations previously defined yields

$$
\begin{array}{r}
\int_{0}^{\infty} \ln \left|S_{h}(j \omega)\right| \mathrm{d} \omega=-\frac{\pi}{2} \lim _{s \rightarrow \infty} s Y_{h}(s) \delta Z_{h}(s) \\
= \begin{cases}-\frac{\pi \delta b_{h}}{2 I_{h}} & \text { for } \delta Z_{h}=\delta b_{h} \\
-\frac{\pi \delta I_{h} \omega_{o}}{2 I_{h}} & \text { for } \delta Z_{h}=\delta I_{h} \frac{\omega_{o} s}{s+\omega_{o}} \\
0 & \text { for } \delta Z_{h}=\frac{\delta k_{h}}{s}\end{cases}
\end{array}
$$

In this way we arrive at a compact result: with the exception of stiffness, negative-valued perturbations cause the area under $\ln \left|S_{h}(j \omega)\right|$ to be positive and vice versa. In other 
words, assistive perturbations cause a net increase in sensitivity, whereas resistive perturbations cause a net decrease 1 . To illustrate these points, Figure 4 shows plots of $\ln \left|S_{h}(j \omega)\right|$ vs. $\omega$ for the different types of perturbation.

2.3 Generating assistive impedance perturbations with an exoskeleton: considerations for control

In (3) the perturbed admittance is represented as the coupling of two dynamical systems: the leg's original admittance $Y_{h}$, and the impedance perturbation $\delta Z_{h}$. Given that we want to design a controller for the coupled system formed by the leg and the exoskeleton, (3) suggests a simple design strategy: substitute $\delta Z_{h}$ with the exoskeleton's port impedance, $Z_{e}(s)$. The task is to design a control to make $Z_{e}(s)$ emulate the behavior of $\delta Z_{h}$ as closely as possible.

The sensitivity transfer function of the coupled system formed by the leg and the exoskeleton is

$S_{h e}(s)=\frac{1}{1+Y_{h}(s) Z_{e}(s)}$

and its loop transfer function is $L_{h e}(s)=Y_{h}(s) Z_{e}(s)$. For the coupled system to emulate assistive (i.e. negative) perturbations of inertia or damping, the Bode sensitivity integral of $S_{h e}(s)$ must be positive. From (8) the only way to accomplish this is by making the gain of $Z_{e}(s)$ negative. In other words, the exoskeleton has to form a positive feedback loop with the human leg, as shown in Figure 3(b).

An important consequence of the gain being negative is that the exoskeleton displays active behavior, i.e. acts as an energy source, which in turn raises the issue of coupled stability. Colgate and Hogan (1988) have shown that a manipulator is guaranteed remain stable, when coupled to an arbitrary passive environment, if the manipulator itself is passive. However, passive behavior can seriously limit performance (Hogan and Buerger, 2006). In the case of an exoskeleton, passive behavior would render it incapable of providing true assistance, at least per the criteria we have outlined in Section 2.1

But then, our requirement is not to ensure stable interaction with every possible passive environment, but only with a certain class of environments, namely those possessing the typical dynamic properties of the human leg. Limiting the set of passive environments with which the exoskeleton is intended to interact allows us, in turn, to use a less restrictive stability criterion. For example, stability can be guaranteed by the Bode criterion for positive feedback:

$\left|-Y_{h}(j \omega) Z_{e}(j \omega)\right|<1$

where $\angle\left(-Y_{h}(j \omega) Z_{e}(j \omega)\right)=-180^{\circ}$

\footnotetext{
1 For stiffness perturbations the area under $\ln \left|S_{h}(j \omega)\right|$ remains constant, meaning that if the sensitivity increases in one frequency range, it will be attenuated in the same proportion elsewhere.
}

In the next section we present the formulation of a stable assistive controller capable of generating a virtual leg admittance with arbitrary values of natural frequency, magnitude peak and, for the integral admittance, DC gain.

\section{Lower-limb assistance by admittance shaping: control design}

\subsection{Specifying a target frequency response for the leg}

We shall base our control design specifications on the human limb's integral admittance, $X_{h}(s) \equiv Y_{h}(s) / s$, expressed in terms of dynamic response parameters:

$X_{h}(s)=\frac{1}{I_{h}\left(s^{2}+2 \zeta_{h} \omega_{n h} s+\omega_{n h}^{2}\right)}$

where $\omega_{n h}$ is the natural frequency of the leg and $\zeta_{h}$ its damping ratio. Our design objective is to make the assisted leg behave in accordance with a target integral admittance model $X_{h}^{d}(s)$, defined as

$X_{h}^{d}(s)=\frac{1}{I_{h}^{d}\left(s^{2}+2 \zeta_{h}^{d} \omega_{n h}^{d} s+\omega_{n h}^{d 2}\right)}$

where $I_{h}^{d}, \omega_{n h}^{d}$ and $\zeta_{h}^{d}$ are, respectively, the desired values of inertia moment, natural frequency and damping ratio. The design specifications are formulated in terms of the following parameter ratios:

$$
\begin{aligned}
R_{\omega} & \equiv \frac{\omega_{n h}^{d}}{\omega_{n h}} \quad \text { (natural frequencies ratio) } \\
R_{M} & \equiv \frac{M_{h}^{d}}{M_{h}} \quad \text { (resonant peaks ratio) } \\
R_{D C} & \equiv \frac{X_{h}^{d}(0)}{X_{h}(0)} \quad \text { (DC gains ratio) }
\end{aligned}
$$

In (15) $M_{h}$ and $M_{h}^{d}$ are, respectively, the magnitude peaks at resonance for $X_{h}(j \omega)$ and $X_{h}^{d}(j \omega)$. Thus our design specifications consist of desired values for $R_{\omega}, R_{M}$ and $R_{D C}$. These specifications are converted into desired values for the dynamic parameters $I_{h}^{d}, \omega_{n h}^{d}, \zeta_{h}^{d}$ by using the following formulas, which are derived in Appendix A

$$
\begin{aligned}
I_{h}^{d} & =\frac{I_{h}}{R_{D C} R_{\omega}^{2}} \\
\omega_{n h}^{d} & =R_{\omega} \omega_{n h} \\
\zeta_{h}^{d} & =\sqrt{\frac{1-\sqrt{1-4 \rho^{2}}}{2}}
\end{aligned}
$$




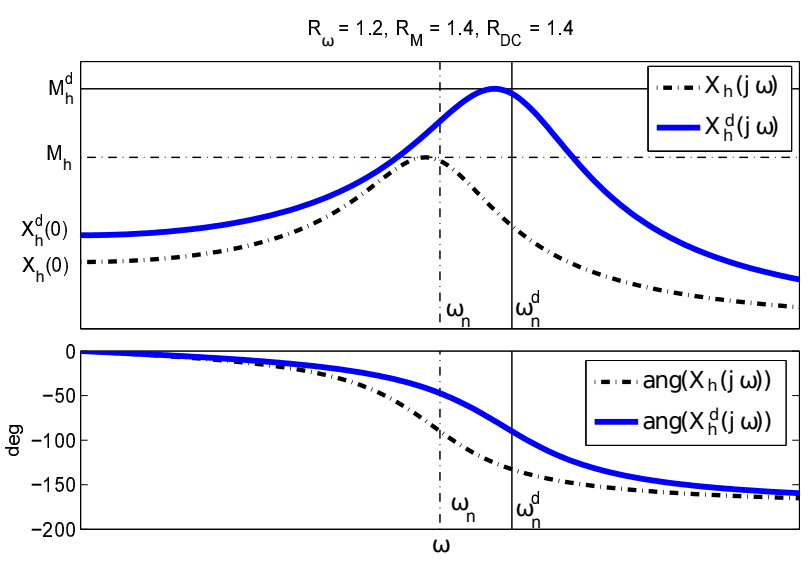

Fig. 5 Frequency responses of the unassisted leg's integral admittance $X_{h}(j \omega)$ and an exemplary target integral admittance $X_{h}^{d}(j \omega)$ with $R_{\omega}=1.2, R_{M}=1.4$ and $R_{D C}=1.4$. The computed parameters for $X_{h}^{d}(j \omega)$ are $I_{h}^{d}=0.4960, \omega_{n h}^{d}=1.2$ and $\zeta_{h}^{d}=0.1989$.

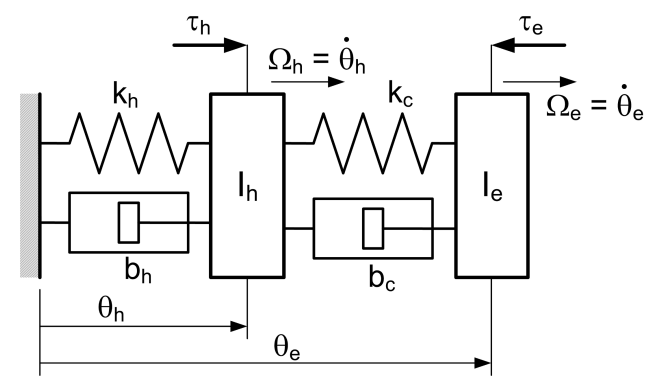

Fig. 6 Linear model of the system formed by the human leg, coupling and exoskeleton mechanism.

where

$\rho=\frac{R_{D C}}{R_{M}} \zeta_{h} \sqrt{1-\zeta_{h}^{2}}$

By way of example, Figure 5 shows a comparison between the frequency responses of the unassisted leg's integral admittance $X_{h}(j \omega)$ and a target integral admittance $X_{h}^{d}(j \omega)$ with specific values of $R_{\omega}, R_{M}$ and $R_{D C}$. This particular target response combines all the possible assistive effects on the leg: increase in natural frequency, effort reduction at resonance, and gravitational support at low frequencies.

Our task is now to design an exoskeleton control capable of making the leg's dynamic response emulate the target $X_{h}^{d}$.

\subsection{Modeling the coupled human-exoskeleton system}

To design the exoskeleton control we shall use the linearized model shown in Figure 6 which represents the human leg coupled to the exoskeleton's arm-actuator assembly (Figure $1(\mathrm{a})$. The inertias of the leg and the exoskeleton are coupled by a spring and damper $\left(k_{c}, b_{c}\right)$ representing the compliance of the leg muscle tissue combined with the compliance of the exoskeleton's thigh brace. In the diagram, ground represents the exoskeleton's hip brace and is assumed to be rigid.

The first design goal is to make the device's mechanism "transparent" when the assistive function is inactive. In other words, the user should feel the effects the mechanism's dynamics (inertia, friction, gravitational effects) as little as possible (Vallery et al, 2009a). This normally requires implementing an inner-loop control, such as an admittance or impedance control, to compensate the reflected inertia, friction and damping of the mechanism, as well as the gravitational torques (Nef et al, 2007). The maximum achievable reduction in reflected inertia is limited by stability boundaries (Colgate and Hogan, 1989a); therefore it is advisable to employ a mechanism design that uses low-inertia components. In the present formulation we will assume that such an inner-loop control is already in place, thereby allowing us to represent the exoskeleton arm as a pure rotational inertia: $Z_{e}(s)=I_{e} s$.

We note that the exoskeleton and the compliant coupling can be represented as second-order impedance given by

$Z_{e c}(s)=I_{e} s+b_{c}+\frac{k_{c}}{s}$

or, equivalently,

$Z_{e c}(s)=I_{e}\left(s+2 \zeta_{e c} \omega_{n, e c}+\frac{\omega_{n, e c}^{2}}{s}\right)$

where $\omega_{n, e c}$ is the natural frequency of the impedance and $\zeta_{e c}$ its damping ratio. The value of $\omega_{n, e c}$ depends on a number of factors like the material of the exoskeleton's thigh coupling and the properties of the subject's thigh tissue. From our system identification studies, values of $\omega_{n, e c}$ in the range of 25 to 50 times the natural frequency of the leg $\left(\omega_{n h}\right)$ are typical for the SMA device. In order to reduce the dimensionality of the analysis somewhat, we assume the impedance (22) to be critically damped, i.e. $\zeta_{e c}=1$. This assumption is warranted because our tests with the SMA have shown $Z_{e c}(s)$ to be actually overdamped, so the criticallydamped assumption is conservative as far as stability is concerned. Keeping our analysis in terms of dimensionless frequencies and damping ratios, we define the following impedance transfer functions:

$$
\begin{aligned}
& Z_{h}(s)=Y_{h}^{-1}(s)=\frac{s^{2}+\zeta_{h} s+1}{s} \\
& Z_{c}(s)=Y_{c}^{-1}(s)=2 I_{e} \omega_{n, e c}\left(\frac{s+\frac{\omega_{n, e c}}{2}}{s}\right) \\
& Z_{e}(s)=Y_{e}^{-1}(s)=I_{e} s
\end{aligned}
$$

We use these impedances to formulate the dynamics equations of the coupled human-exoskeleton system of Figure 6 


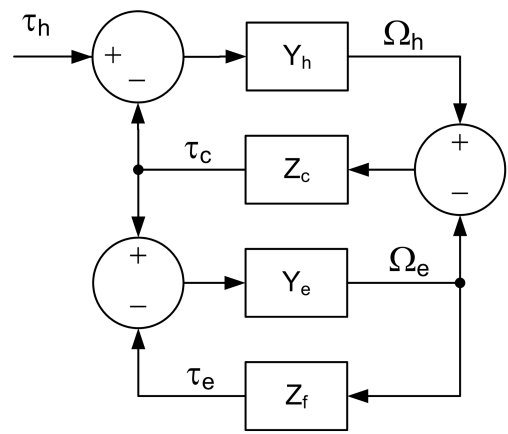

(a)

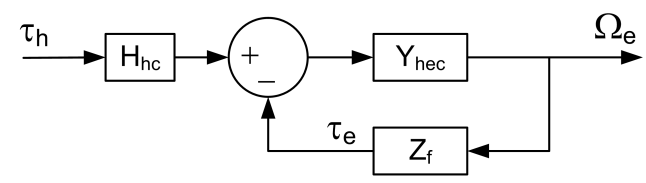

(b)

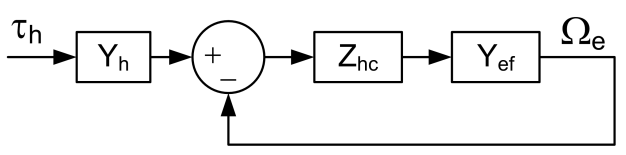

(c)

Fig. 7 Block diagrams of the system formed by the human leg, coupling, exoskeleton mechanism and interaction controller. (a) Block diagram derived from equations 26 to 29. (b) Equivalent block diagram for control design. The transfer function $Y_{\text {hecf }}(s)=\Omega_{e}(s) / \tau_{h}(s)$ represents the admittance of the human leg assisted by the exoskeleton. (c) Equivalent block diagram for stability robustness analysis.

in the Laplace domain (the derivation is omitted due to space limitations):

$$
\begin{aligned}
\Omega_{h} & =Y_{h}\left(\tau_{h}-\tau_{c}\right) \\
\tau_{c} & =Z_{c}\left(\Omega_{h}-\Omega_{e}\right) \\
\Omega_{e} & =Y_{e}\left(\tau_{c}-\tau_{e}\right)
\end{aligned}
$$

where $\tau_{c}$ is the interaction torque between the leg and the exoskeleton (exerted through the coupling) and $\tau_{e}$ is the torque generated by a feedback compensator $Z_{f}(s)$ :

$\tau_{e}=Z_{f} \Omega_{e}$

$Z_{f}(s)$ embodies the exoskeleton's assistive action. It should be noted that, although the compensator takes in angular velocity feedback, $Z_{f}(s)$ may contain derivative or integral terms. Therefore the physical control implementation may involve feedback of angular acceleration or angular position. Another observation is that, although the torque generated by the control is $\tau_{e}$, the actual torque exerted on the leg by the exoskeleton is $\tau_{c}$.

\subsection{Design of the assistive control}

Using equations (26), (27), (28) and (29), we represent the coupled leg-exoskeleton system as the block diagram of Figure 7(a). The aim of the assistive control is to make the dynamic response of this system such that it matches the frequency response of the target integral admittance $X_{h}^{d}(s)$. Our control design method is a two-step procedure:

- Design of an angle feedback compensator to achieve the target DC gain (stiffness and gravity compensation).

- Design of an angular acceleration feedback compensator to achieve the target natural frequency and target resonant peak. This second compensator is designed using a pole placement technique and has to ensure the stability of the coupled system.

Decoupling the DC gain problem from the other two is valid because, as can be seen on Figure 2, the DC gain is only affected by a stiffness perturbation, which is easily implemented via angle feedback. The same figure suggests that the natural frequency target could be achieved by either angle feedback (stiffness perturbation) or angular acceleration feedback (inertia perturbation). By choosing angular acceleration feedback we avoid creating a conflict with the DC gain objective, which depends exclusively on angle feedback. Furthermore, we will show that employing an angular acceleration feedback compensator with sufficient degrees of freedom allows us to achieve the natural frequency and resonant peak targets simultaneously.

\subsubsection{Feedback compensator for target DC gain}

The design of the compensator for target DC gain is a simple application of the dynamics of the coupled system in the static (zero frequency) case. From Figure 6, torque balance on the human leg's inertia $I_{h}$ yields

$k_{h} \theta_{h}=\tau_{h}-\tau_{c}$

Torque balance on the exoskeleton's inertia $I_{e}$ yields

$\tau_{c}-\tau_{e}=0$

Because the objective is to compensate for the stiffness and gravitational torque acting on the leg, the assistive torque is provided by a virtual spring:

$\tau_{e}=k_{D C} \theta_{e}$

Assuming the coupling to have sufficient stiffness so that $\theta_{e} \simeq \theta_{h}$, from 30 the net muscle torque becomes

$\tau_{h}=k_{h} \theta_{h}-k_{D C} \theta_{e} \simeq\left(k_{h}-k_{D C}\right) \theta_{h}$ 
To determine the virtual spring stiffness $k_{D C}$ we refer to Appendix A Equation (79) defines an intermediate target integral admittance $X_{h, D C}(s)$ embodying the DC gain specification. Maintaining the assumption $\theta_{e} \simeq \theta_{h}$ we note that $X_{h, D C}(s)$ can be implemented by adding the virtual spring to the human leg's impedance. Thus an alternative definition is

$\widehat{X}_{h, D C}(s)=\frac{1}{I_{h} s^{2}+2 I_{h} \zeta_{h} \omega_{n h} s+I_{h} \omega_{n h}^{2}+k_{D C}}$

Making $\widehat{X}_{h, D C}(0)=X_{h, D C}(0)$ yields

$I_{h} \omega_{n h}^{2}+k_{D C}=I_{h} \omega_{n h, D C}^{2}$

But from we have $\omega_{n h, D C}^{2}=R_{D C}^{-1} \omega_{n h}^{2}$. Thus we obtain the stiffness and gravity compensation gain as:

$k_{D C}=I_{h} \omega_{n h}^{2}\left(R_{D C}^{-1}-1\right)$

The angular position feedback (32) with the computed value of $k_{D C}$ generates the following closed-loop exoskeleton admittance:

$Y_{e, D C}=\frac{Y_{e}}{1+\frac{k_{D C}}{s} Y_{e}}$

Clearly, for a DC gain specification of $R_{D C}>1$ we have $k_{D C}<0$, i.e. positive feedback of the angular position. In consequence, the closed-loop exoskeleton admittance has a pole at $s=+\sqrt{k_{D C} I_{e}^{-1}}$, which makes the isolated exoskeleton unstable. However, the coupled system formed by the leg and the exoskeleton will be stable as long as the virtual stiffness coefficient of the assisted leg remains positive. A detailed analysis of the stability of the coupled system with DC gain compensator is given in the next section.

\subsubsection{Loop transfer function of the coupled human limb and exoskeleton}

With the compensator for the target DC gain already in place, the forthcoming analysis will focus on the target admittance for the assisted leg, given by $Y_{h}^{d}(s)=s X_{h}^{d}(s)$. The objective is now to design a compensator capable of increasing the natural frequency of the leg as well as the magnitude peak of its admittance. This is also the more involved part of our control design, since we need to design for both performance and stability. Although, strictly speaking, we want to control the relationship between the human muscle torque $\tau_{h}$ and the leg's angular velocity $\Omega_{h}$ as to match $Y_{h}^{d}(s)$, our design will focus on the transfer function relating $\tau_{h}$ to the exoskeleton angular velocity $\Omega_{e}$, the reason being that we only have a practical way of measuring $\Omega_{e}$. This is acceptable under the assumption that the coupling is sufficiently rigid and therefore $\Omega_{e} \simeq \Omega_{h}$.

We begin by substituting $Y_{e}(s)$ with $Y_{e, D C}(s)$ in Figure 7(a) and converting the block diagram to a form suitable for analysis using the system's loop transfer function. Figure 7(b) shows the equivalent block diagram (derivation omitted for brevity), which contains the following transfer functions:

$Y_{h e c}(s)=\frac{Z_{h}+Z_{c}}{Z_{h} Z_{e, D C}+Z_{c} Z_{e, D C}+Z_{c} Z_{h}} \equiv \frac{N_{h e c}(s)}{D_{h e c}(s)}$

where $Z_{e, D C}=Y_{e, D C}^{-1}$, and

$H_{h c}(s)=\frac{Z_{c}}{Z_{h}+Z_{c}}=\frac{Z_{c}}{N_{h e c}(s)}$

From Figure 7(b) we derive the transfer function relating the human torque to the encoder angular velocity:

$Y_{h e c f}(s)=\frac{\Omega_{e}(s)}{\tau_{h}(s)}=\frac{Z_{c}}{D_{h e c}+Z_{f} N_{h e c}}$

$Y_{h e c f}(s)$ is very important in our analysis because it represents the admittance of the human leg assisted by the exoskeleton. From linear feedback control theory, the dynamic response properties of $Y_{\text {hecf }}(s)$ are determined mainly by its characteristic polynomial. Therefore we shall formulate the design of the compensator $Z_{f}(s)$ as a pole placement problem: namely, to make the dominant poles of $Y_{\text {hecf }}(s)$ match the poles of the target admittance $Y_{h}^{d}(s)$. Our design will use the standard tools of root locus and Bode stability applied to the loop transfer function of $Y_{\text {hecf }}(s)$.

From Figure 7(b), we define the loop transfer function, $L_{\text {hecf }}(s)$, as a ratio of monic polynomials obeying

$K_{L} L_{h e c f}(s)=Z_{f}(s) Y_{h e c}(s)$

where $K_{L}$ is the loop gain. Success in the design of the feedback compensator $Z_{f}(s)$ requires ensuring that the feedback loop formed by $Z_{f}(s)$ and $Y_{h e c}(s)$ is stable. Given that $Y_{h e c}(s)$ already contains a positive feedback loop of the angular position (through $Z_{e, D C}$ ), we want to analyze its stability properties before designing $Z_{f}(s)$. For the forthcoming analysis we shall use the dimensionless moment of inertia of the SMA arm and actuator assembly, $I_{e}=0.00702$. We begin by writing the impedances in (38) in terms of polynomial ratios and gains: 


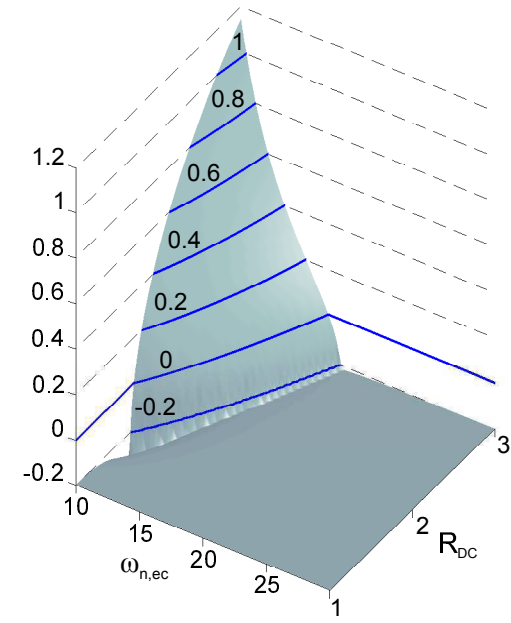

Fig. 8 Contour plot showing the real part of the dominant poles of $Y_{h e c}(s)$ (equation 43) as a function of the DC gain ratio $R_{D C}$ and the coupling's natural frequency, $\omega_{n, e c}$.

$$
\begin{aligned}
& Z_{h}(s)=\frac{s^{2}+\zeta_{h} s+1}{s} \quad \equiv \frac{N_{h}(s)}{s} \\
& Z_{c}(s)=2 I_{e} \omega_{n, e c}\left(\frac{s+\frac{\omega_{n, e c}}{2}}{s}\right) \equiv z_{c o} \frac{N_{c}(s)}{s} \\
& Z_{e, D C}(s)=I_{e}\left(\frac{s^{2}+\frac{k_{D C}}{I_{e}}}{s}\right) \equiv I_{e} \frac{N_{e}(s)}{s}
\end{aligned}
$$

This, in turn, allows us to write $Y_{h e c}(s)$ as the following ratio of polynomials:

$Y_{h e c}(s)=\frac{s\left(N_{h}+z_{c o} N_{c}\right)}{I_{e} N_{e} N_{h}+z_{c o} N_{e} N_{c}+z_{c o} N_{h}}$

From inspection of (42) and 43) we find that $Y_{h e c}(s)$ has four poles and three zeros, including one zero at the origin.

Figure 8 shows contour plots of the real part of the dominant poles of $Y_{h e c}(s)$ as a function of the DC gains ratio $R_{D C}$ and the natural frequency of the coupling $\omega_{n, e c}$. These poles are located near the poles of the human leg's admittance, $-0.2 \pm 0.980 j$. We can observe that, for most values of $R_{D C}$ and $\omega_{n, e c}$, the dominant poles' real part is constant and equal to -0.2 , i.e. the dominant poles are stable. Only for combinations of very low natural frequency of the coupling and high DC gain ratios do the dominant poles cross over to the right-hand side of the complex plane (RHP). For now we will maintain the assumption that the $R_{D C}$ specification is such that does not violate the stability of $Y_{h e c}$. Ensuring that $Y_{h e c}$ has no RHP or imaginary poles guarantees the existence of a range of negative loop gains $K_{L}$ for which the closed-loop transfer function $Y_{\text {hecf }}(s)$ is stable as well.
3.3.3 Feedback compensator for target values of natural frequency and resonant peak: design by pole placement

In order to explain the derivation of the feedback compensator $Z_{f}(s)$ for natural frequency and resonant peak targets, we will use a specific design example involving the Honda SMA device. We set forth the following design specifications: $R_{\omega}=1.2, R_{M}=1.3$ and $R_{D C}=1.1$, which in turn yield the following parameter values for the target integral admittance (13): $I_{h}^{d}=0.631, \omega_{n h}^{d}=1.2$ and $\zeta_{h}^{d}=0.1673$. Given these values, the desired locations of the dominant poles are

$p_{h}^{d}=-\sigma_{h}^{d}+j \omega_{d h}^{d}=-0.201+1.183 j$
$\bar{p}_{h}^{d}=-\sigma_{h}^{d}-j \omega_{d h}^{d}=-0.201-1.183 j$

where

$$
\begin{aligned}
\sigma_{h}^{d} & =\zeta_{h}^{d} \omega_{n h}^{d} \\
\omega_{d h}^{d} & =\omega_{n h}^{d} \sqrt{1-\zeta_{h}^{d 2}}
\end{aligned}
$$

The gain of the feedback compensator for target DC gain is computed with 36, yielding $k_{D C}=-0.0909$.

In Section 2.1 we showed that, in the ideal case, an increase in natural frequency can be accomplished by compensating the second-order system's inertia. This suggests that our compensator should include positive acceleration feedback in some form. Although pure acceleration feedback will not satisfy our design requirements, it is nonetheless instructive to examine its behavior. Thus we define the provisional compensator

$\widehat{Z}_{f}(s) \equiv-I_{c} s$

We shall refer to $I_{c}$ as the inertia compensation gain. Then we write

$\widehat{Z}_{f}(s) Y_{h e c}(s)=-\frac{I_{c}}{I_{e}} s L_{h e c}$

where $L_{h e c}(s)$ is a ratio of monic polynomials possessing the same poles and zeros as $Y_{h e c}(s)$. From (46) the loop gain for pure acceleration feedback as

$\widehat{K}_{L}=-\frac{I_{c}}{I_{e}}$

Figure 9 (a) shows the root loci of $s L_{h e c}(s)$ for both positive and negative feedback. Because all the coefficients of $s L_{h e c}(s)$ are fixed, the shape of the root loci is constant and the only variable parameter we have is the loop gain $\widehat{K}_{L}$. 


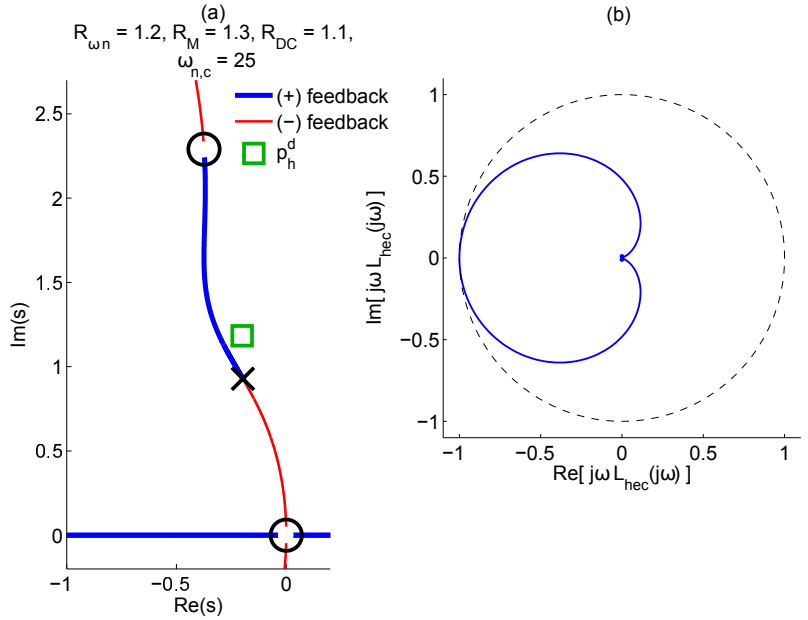

Fig. 9 (a) Root loci (detail) of $s L_{h e c}(s)$ for positive and negative feedback. The positive-feedback root locus misses the target location for the dominant closed-loop pole, $p_{h}^{d}$. (b) Nyquist diagram for $-s L_{h e c}(s)$.

Therefore, it will be only by exception that the positivefeedback root locus passes through the target location for the dominant pole, $p_{h}^{d}$.

However, the most serious limitation of the compensator is its stability range. For positive feedback, the coupled system's stability is determined by the stability margins of $-s L_{h}$ Because $-s L_{h e c}(s)$ has four poles and four zeros, at $s=$ $+j \infty$ we have $\left|-s L_{h e c}(s)\right|=1$ and $\angle\left(-s L_{h e c}(s)\right)=180^{\circ}$. In other words, closing a feedback loop with $-s L_{h e c}(s)$ puts the system is at its instability threshold. This condition is patent in the Nyquist diagram of Figure 9 (b). Noting that in the Nyquist diagram $-s L_{h e c}(s)$ implies a loop gain $\widehat{K}_{L}=$ 1 , from 477 the limiting value for the inertia compensation gain is

$I_{c}=I_{e}$

Thus we see that, for pure positive acceleration feedback, the best that the exoskeleton can do before causing instability is to cancel its own inertia, but none of human leg's inertia.

In order to overcome the limitations of pure positive acceleration feedback, we add a pair of complex conjugate poles $-\sigma_{f} \pm j \omega_{d, f}$ to the compensator. To this end we define the second-order filter

$H_{f}(s) \equiv \frac{\sigma_{f}^{2}+\omega_{d, f}^{2}}{s^{2}+2 \sigma_{f} s+\sigma_{f}^{2}+\omega_{d, f}^{2}}$

Our proposed feedback compensator model is then

$Z_{f}(s) \equiv-I_{c} s H_{f}(s)$ where $\sigma_{f}$ and $\omega_{d, f}$ are parameters to be determined. With $Z_{f}(s)$ thus defined, and recalling 41, the loop transfer function now becomes

$L_{h e c f}(s)=\frac{s L_{h e c}(s)}{s^{2}+2 \sigma_{f} s+\sigma_{f}^{2}+\omega_{d, f}^{2}}$

Thus given a loop gain $K_{L}<0$ that meets the design requirements, the inertia compensation gain is obtained as

$I_{c}=-\frac{K_{L} I_{e}}{\sigma_{f}^{2}+\omega_{d, f}^{2}}$

The rationale behind our compensator model is that $\sigma_{f}$ and $\omega_{d, f}$ provide two degrees of freedom with which to shape the positive-feedback root locus of $L_{\text {hecf }}(s)$. Shaping the root locus pursues two different objectives:

- Making the root locus pass through the target locations of the dominant poles, $p_{h}^{d}$ and $\bar{p}_{h}^{d}$ or as close to them as possible. This ensures that, with an appropriate gain $I_{c}$, the system's closed-loop transfer function $Y_{\text {hecf }}(s)$ (Figure 7(b) will have poles at or near $p_{h}^{d}$ and $\bar{p}_{h}^{d}$.

- Maximizing the stability margins of $Y_{h e c f}(s)$ as to ensure that the design solution provided by $\sigma_{f}, \omega_{d, f}$ and $(s) . I_{c}$ is stable. It must be noted that, with positive feedback, the root locus is guaranteed to enter the RHP as $K_{L} \rightarrow-\infty$. Therefore it is in general possible that, while two of the closed-loop poles of $Y_{h e c f}(s)$ satisfy $s=p_{h}^{d}$, any of the remaining poles may be in the RHP. By maximizing the stability margins we seek to avoid this risk.

The inclusion of two poles in (51) also ensures that the frequency response of $L_{\text {hecf }}(s)$ rolls off at high frequencies, thereby making the system less sensitive to feedback of high-frequency noise.

Our compensator design requires solving a pole placement problem, namely finding values of $\sigma_{f}, \omega_{d, f}$ and $I_{c}$ such that $Y_{\text {hecf }}(s)$ has poles at $p_{h}^{d}$ and $\bar{p}_{h}^{d}$. We refer to $\left\{\sigma_{f}\right.$, $\left.\omega_{d, f}, I_{c}\right\}$ as a candidate solution. Only when this solution guarantees the stability of the coupled system can it be considered a valid compensator design. Solutions for the pole placement problem can be found by applying the properties of the positive-feedback root locus as described below.

- Phase property: for $s=p_{h}^{d}$ the phase $\Phi$ of $L_{h e c f}(s)$ must be equal to zero. We express this condition as

$\Phi=\Phi\left(\sigma_{f}, \omega_{d, f}, p_{h}^{d}\right)=\angle L_{h e c f}\left(p_{h}^{d}\right)=0$

which yields a range of solutions for $\sigma_{f}$ and $\omega_{d, f}$.

- Gain property: for $s=p_{h}^{d}$ the loop gain $K_{L}$ satisfies

$$
K_{L}=K_{L}\left(\sigma_{f}, \omega_{d, f}, p_{h}^{d}\right)=\frac{-1}{\left|L_{h e c f}\left(p_{h}^{d}\right)\right|}
$$



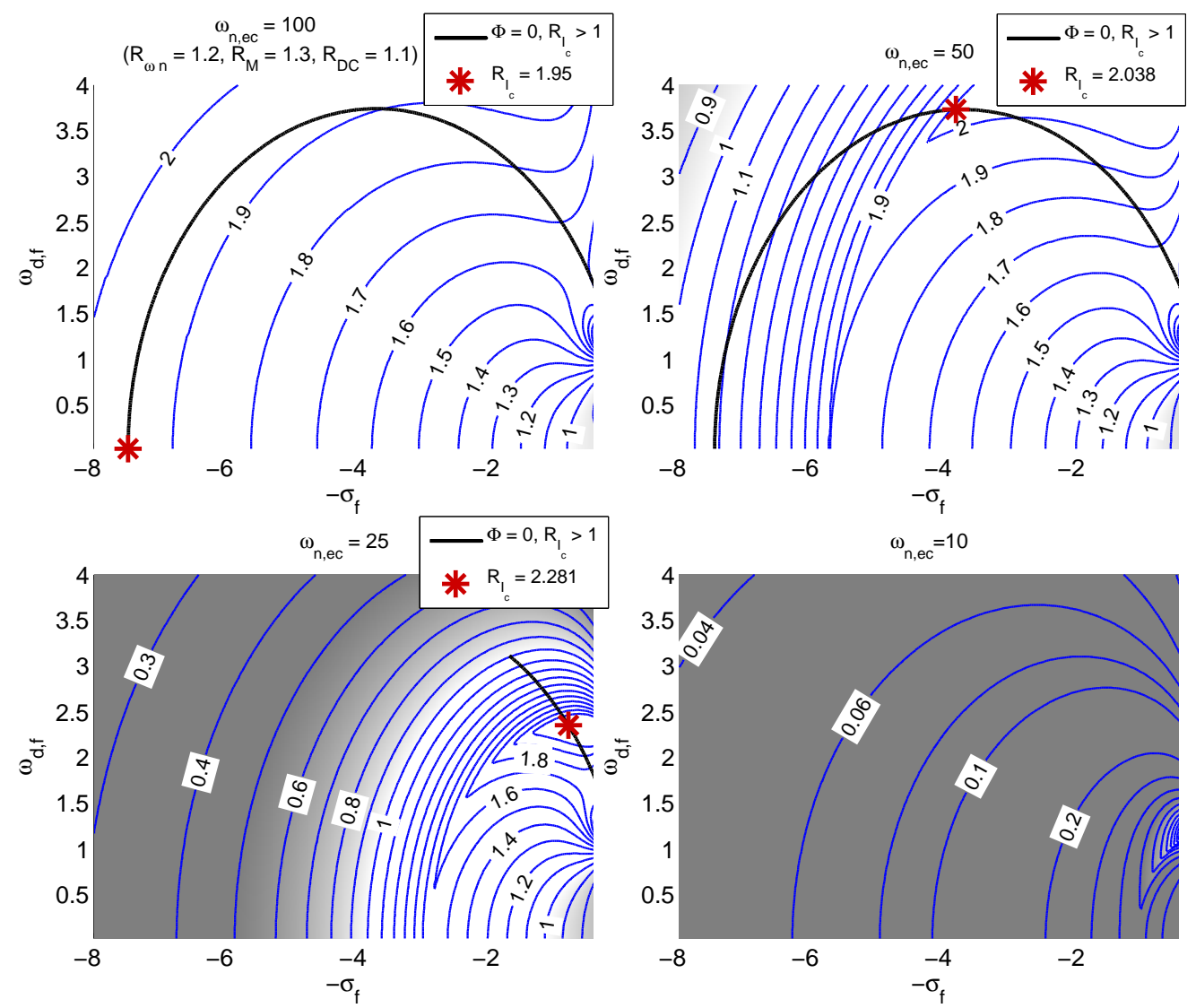

Fig. 10 Phase property and gain margins of the coupled system formed by the human limb, the exoskeleton and the compensator with positive feedback. The target dominant pole of the system's response is $p_{h}^{d}=-0.201 \pm 1.183 j$. Each graph represents a different value of the coupling stiffness, given by $\omega_{n, e c}$. Contour plots represent the ratio of inertia compensation gains $R_{I_{c}}$ [58) as a function $\sigma_{f}$ and $\omega_{d, f}$. Gray regions correspond to the case where $R_{I_{c}} \leq 1$, i.e. where the coupled system is unstable; white regions represent the stable case $R_{I_{c}}>1$. Thick curves labeled " $\Phi=0, R_{I_{c}}>1$ " represent stable solutions to the pole placement problem, i.e. combinations $\left\{\sigma_{f}, \omega_{d, f}, I_{c}\right\}$ that generate a closed-loop pole at $s=p_{h}^{d}$ by satisfying the phase condition $\Phi\left(\sigma_{f}, \omega_{d, f}, p_{h}^{d}\right)=0$, and also meet the stability condition $R_{I_{c}}>1$. For every such curve there is an optimal solution, marked by an asterisk $(*)$, that corresponds to a maximum for $R_{I_{c}}$, i.e. a maximal gain margin. Said solution is found by solving the optimization problem [59. (Note: the lowest compliance case, $\omega_{n, e c}=10$, yielded no stable solutions.)

The formulas for computing $\Phi$ and $K_{L}$ are given in Appendix B. Given a solution pair $\left\{\sigma_{f}, \omega_{d, f}\right\}$ and the value of $K_{L}$ resulting from (54), the inertia compensation gain $I_{c}$ is computed using 52 .

Now, it must be kept in mind that the positive-feedback root locus properties by themselves do not guarantee the stability of the coupled system. $L_{\text {hecf }}(s)$ has six poles in total; conditions (53) and (54) guarantee that two of the closedloop poles are stable since $p_{h}^{d}$ and $\bar{p}_{h}^{d}$ lie in the left-hand half of the complex plane, but they say nothing about the stability of the remaining four poles. The stability of the candidate solution $\left\{\sigma_{f}, \omega_{d, f}, I_{c}\right\}$ depends on the value of $I_{c}$. If we define $I_{c, M}$ as the inertia compensation gain that puts the closed-loop system at the threshold of instability for given values of $\sigma_{f}$ and $\omega_{d, f}$, the stability condition is

$R_{I_{c}} \equiv \frac{I_{c, M}}{I_{c}}>1$
In order to compute $R_{I_{c}}$, the loop gain at the instability threshold is obtained as

$K_{L, M}=\frac{-1}{\left|L_{h e c f}\left(j \omega_{M}\right)\right|}$

where

$\omega_{M}=\omega \mid \angle\left(-L_{h e c f}(j \omega)\right)=-180^{\circ}$

From (52) the ratio of inertia compensation gains can be computed simply as

$R_{I_{c}}=\frac{I_{c, M}}{I_{c}}=\frac{K_{L, M}}{K_{L}}$

$R_{I_{c}}$ constitutes a stability margin; to be precise, a gain margin. Therefore it will play an important role in the design of the compensator. 


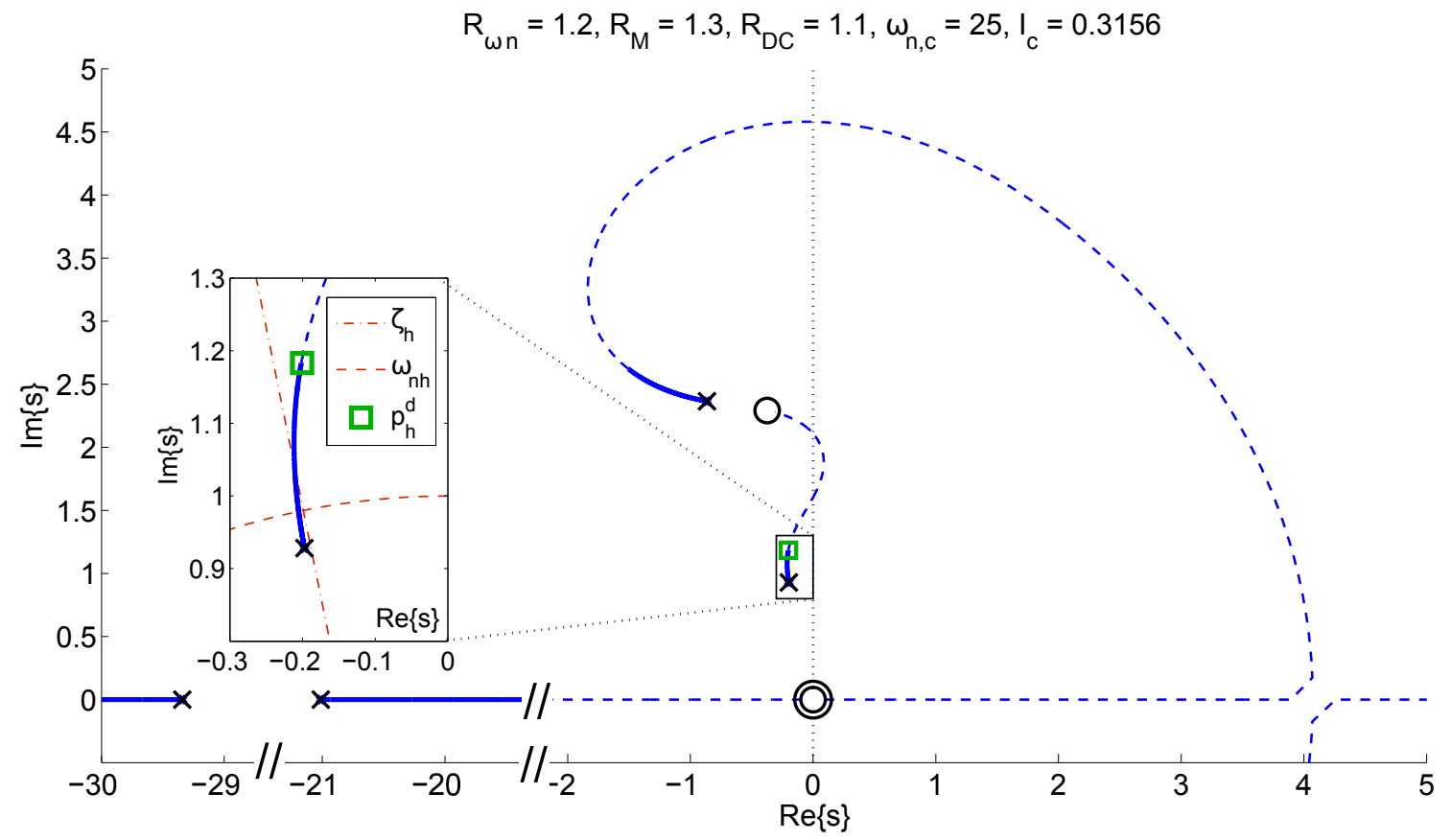

Fig. 11 Human-exoskeleton system with feedback compensator optimized for the design specifications of Section 3.3 .3 Plot shows the positivefeedback root locus of the loop transfer function $L_{h e c f}(s)$. Coupling stiffness is $\omega_{n, e c}=25$. Dashed portions of the root locus correspond to loop gains greater in magnitude than the computed value $K_{L}$ 93. The inset shows how the root locus passes exactly through the target location of the dominant pole, $p_{h}^{d}$; curves corresponding to the damping ratio $\zeta_{h}$ and the natural frequency $\omega_{n h}$ of the unassisted leg are shown for comparison.

At this point we need to consider that the values of the system's parameters involve considerable uncertainty, especially in the case of the human leg and the coupling. Aside from its implications on performance, parameter uncertainty poses the risk of instability. Thus the physical coupled system could be unstable even though the compensator is theoretically stabilizing. To minimize that risk, we propose formulating the design of the compensator as a constrained optimization problem: given the target dominant pole $s=$ $p_{h}^{d}$, to find a combination $\left\{\sigma_{f}, \omega_{d, f}, I_{c}\right\}$ that maximizes the inertia compensation gains ratio $R_{I_{c}}$ while preserving the phase condition 53.

To test the feasibility of this approach, we computed the values of $\Phi\left(\sigma_{f}, \omega_{d, f}, p_{h}^{d}\right)$ and $R_{I_{c}}$ for a matrix of values of $\sigma_{f}$ and $\omega_{d, f}$ and with different values of coupling stiffness $\omega_{n, e c}$ as a parameter. The results are shown in Figure 10. Contour plots represent constant values of $R_{I_{c}}$; values of $R_{I_{c}}$ greater than unity (indicated by white regions in the plot) represent a stable coupled system. The thicker curves labeled " $\Phi=0, R_{I_{c}}>1$ " are loci of stable solutions $\left\{\sigma_{f}, \omega_{d, f}, I_{c}\right\}$ to the pole placement problem, i.e. solutions that simultaneously satisfy the phase condition $\Phi\left(\sigma_{f}, \omega_{d, f}, p_{h}^{d}\right)$ 0 and the stability condition $R_{I_{c}}>1$. As to why the compensator is capable of generating stable solutions, the general principle is given in Section 3.4 with the aid of an example.
Inspection of the contour plots for $R_{I_{c}}$ shows that for each stable solution curve it is possible to find a maximum for $R_{I_{c}}$. We also notice that the solution curves tend to contract and finally disappear as $\omega_{n, e c}$ decreases, which shows that $\omega_{n, e c}$ has an important influence on the stability robustness of the assistive control.

Thus we formulate the feedback compensator design problem as follows: given a target dominant pole $p_{h}^{d}$, find

$$
\begin{array}{cl}
\max _{\left\{\sigma_{f}, \omega_{d, f}\right\}} & R_{I_{c}}^{2}\left(\sigma_{f}, \omega_{d, f}, p_{h}^{d}\right) \\
\text { subject to } & \Phi\left(\sigma_{f}, \omega_{d, f}, p_{h}^{d}\right)=0
\end{array}
$$

The complete design procedure of the assistive control for admittance shaping is summarized thus:

1. Formulate the design specifications $R_{\omega}, R_{M}$ and $R_{D C}$.

2 . With the DC gain specification $R_{D C}$, compute the angular position feedback gain $k_{D C}$ using (36).

3. Compute the target admittance parameters $\omega_{n h}^{d}$ and $\zeta_{h}^{d}$ using (18) and (19).

4. Obtain the dominant pole of the target admittance as $p_{h}^{d}=-\sigma_{h}^{d}+j \omega_{d h}^{d}$ using (44).

5. Obtain the parameters $\left\{\sigma_{f}, \omega_{d, f}\right\}$ of the feedback compensator $Z_{f}(s)$ (49), 50 by performing the constrained optimization 59.

6. With $\left\{\sigma_{f}, \omega_{d, f}\right\}$ obtain the the loop gain $K_{L}$ using (93) and the inertia compensation gain $I_{c}$ using (52). 

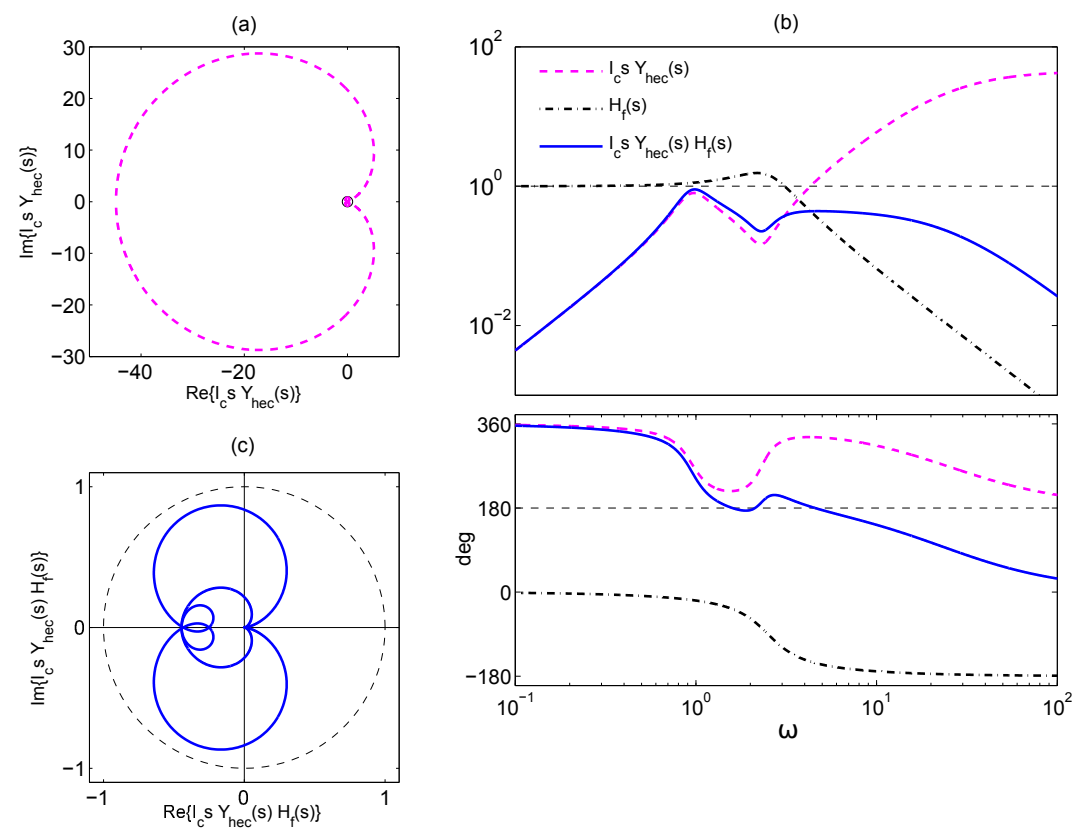

Fig. 12 Coupled human-exoskeleton system with feedback compensator: stability analysis. (a) Nyquist diagram for the open-loop transfer function with pure positive acceleration feedback: the diagram encircles -1, showing that the coupled system is unstable. (b) Frequency responses of the open-loop transfer function before and after adding the second-order filter $H_{f}(s)$. The filter causes the response magnitude to roll off and be less than unity at all frequencies. (c) Nyquist diagram for the open-loop transfer function with added second-order filter. No encirclement of -1 occurs; therefore the coupled system is stable.
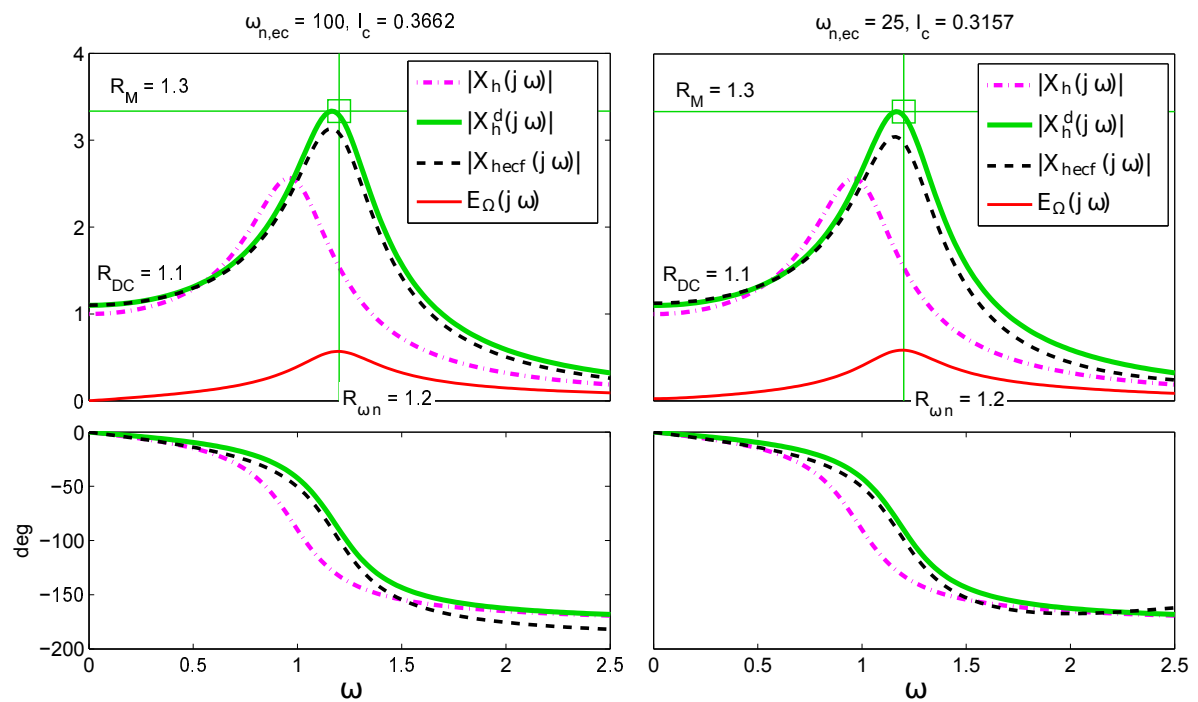

Fig. 13 Frequency response of the integral admittance of the human-exoskeleton system with feedback compensator $\left(X_{\text {hecf }}(s)\right)$. Magnitude ratio and phase plots are shown for $\omega_{n, e c}=100$ and $\omega_{n, e c}=25$. Frequency responses of the integral admittance of the unassisted leg $\left(X_{h}(s)\right)$ and the target integral admittance $\left(X_{h}^{d}(s)\right)$ are included for comparison.

3.4 Assistive control for admittance shaping: design examples

\subsubsection{Feedback compensator designs}

We performed the above procedure for the design specifications given at the beginning of Section 3.3.3. Compensator designs were generated for different values of coupling stiff- ness. The constrained optimization (59) was performed using the fmincon function in Matlab (The Mathworks, Natick, MA, USA) with the trust-region reflective algorithm (More and Sorensen, 1983). The initial conditions employed were $\sigma_{f}=4.0$ and $\omega_{d, f}=2.0$ and the termination tolerance for the variables was set to $10^{-8}$. The algorithm was able to find stable solutions for the pole placement problem; the resulting values for the feedback compensator parameters and the 
Table 1 Feedback compensator parameters and associated inertia compensation gains ratio

\begin{tabular}{llll}
\hline Parameter & $\omega_{n, e c}=100$ & $\omega_{n, e c}=50$ & $\omega_{n, e c}=25$ \\
\hline$\sigma_{f}$ & 7.4877 & 3.8427 & 0.8622 \\
$\omega_{d, f}$ & $3.98 \times 10^{-5}$ & 3.7292 & 2.3513 \\
$I_{c}$ & 0.3662 & 0.3627 & 0.3148 \\
\hline$R_{I_{c}}$ & 1.950 & 2.038 & 2.281 \\
\hline
\end{tabular}

corresponding $R_{I_{c}}$ values are shown in Table 1 The solutions are also shown graphically in Figure 10, each asterisk (*) represents the optimal values of $\sigma_{f}, \omega_{d, f}$ and $R_{I_{c}}$ for a particular stiffness value $\omega_{n, e c}$.

Figure 11 shows the positive-feedback root locus of $L_{\text {hecf }}(s)$ for the coupling with $\omega_{n, e c}=25$. This figure illustrates the fact that it is possible to find compensator solutions that achieve the pole placement objective, despite the fact that positive feedback tends to destabilize the coupled system, as indicated by the incursions of the root locus into the RHP as $K_{L} \rightarrow-\infty$.

\subsubsection{Stability of the coupled system}

The stabilizing effect of the second-order filter (49) can be understood by comparing once more against pure positive acceleration feedback. Taking the case $\omega_{n, e c}=25$ in Table [1. it is clear that, if we attempt pure positive acceleration feedback using the prescribed gain $I_{c}$, the coupled system will be unstable. Figure 12 a) shows the Nyquist diagram of the corresponding open-loop transfer function, $I_{c} s Y_{h e c}(s)$. This is the same diagram as Figure 9 b) but scaled in magnitude; the plot encircles the critical point -1 as a result. The Bode plot of $I_{c} s Y_{h e c}(s)$ in Figure 12 b) shows that the magnitude tends to a maximum $\gg 1$ as $\omega \rightarrow+\infty$. However, this effect is counteracted by the frequency rolloff characteristic of the filter $H_{f}(s)$. Adding the filter results in an open-loop transfer function $I_{c} s Y_{h e c}(s) H_{f}(s)$ of which the magnitude rolls off as well. As a consequence, the magnitude never exceeds a value of 1 , thereby avoiding any encirclement of -1 .

Figure 12 (c) shows the Nyquist diagram for the coupled system with the second-order filter in place. It can be seen that the solution is robust to both phase variations and gain variations. The only limitation is that the gain margin is finite, whereas the phase margin is infinite. Thus in principle it is possible for the coupled system to maintain stability in spite of discrepancies between the system's model and the actual properties of the physical leg and exoskeleton.

\subsubsection{Performance of the coupled system: frequency response}

Our design goal was to make the dynamic response of the exoskeleton-assisted leg match the integral admittance model $X_{h}^{d}(s)$ 13) as closely as possible. Figure 13 shows a comparison between the frequency response of the coupled system's integral admittance $X_{\text {hecf }}(s)$ and the response of the model $X_{h}^{d}(s)$. The frequency response of the unassisted leg (modeled by $X_{h}(s)$ ) is shown as well for reference. It can readily be seen that the response of the coupled system closely matches that of the model despite the differences of order among the transfer functions $\left(X_{h}^{d}(s)\right.$ only has two poles, whereas $X_{\text {hecf }}(s)$ has six poles and four zeros.)

In the next section we examine the stability robustness $s$ ) of the exoskeleton's control to variations in the parameters of the coupled system.

\section{Stability robustness of the exoskeleton control}

\subsection{Robustness to stiffness parameters}

The present analysis assumes the exoskeleton model $Z_{e}$ to be accurate and focuses on two system parameters that are particularly difficult to identify, the stiffness of the human leg's joint and the stiffness of the coupling. The passive stiffness of the hip joint can be estimated with reasonable accuracy under highly controlled conditions (Fee and Miller, 2004). However, hip impedance also has reflexive components due to muscle activation (Schouten et al, 2008). The stiffness of the coupling between the leg and the exoskeleton, on its part, depends not only on the thigh brace but also on the compliance of the thigh tissue, which is a highly uncertain quantity.

We begin by converting the system's block diagram in Figure 7(a) to the equivalent form of Figure 7(c). In this diagram, the parameters of the human limb and the coupling are bundled together in the transfer function $Z_{h c}$, defined as

$Z_{h c}(s)=Y_{h c}^{-1}(s)=\left(Y_{h}+Y_{c}\right)^{-1}$

We shall use the transfer function thus defined to analyze the effects of uncertainties in the stiffness of the human leg's joint and the stiffness of the coupling. The other transfer function in the feedback loop, $Y_{e f}$, combines the parameters of the exoskeleton and the feedback compensator, and is defined as

$Y_{e f}(s)=Z_{e f}^{-1}(s)=\left(Z_{e}+Z_{f}\right)^{-1}$

We shall consider the exoskeleton-compensator system $Y_{e f}(s)$ to provide robust stability if it guarantees the sta- 
bility of the closed-loop system of Figure $7(\mathrm{c})$ for a reasonably large range of variations in the uncertain parameters. To this end we define the system's nominal closed-loop transfer function, $T_{h e c f}(s)$ as

$T_{h e c f}(s)=\frac{Z_{h c} Y_{e f}}{1+Z_{h c} Y_{e f}}=\frac{1}{1+Y_{h c} Z_{e f}}$

The perturbed closed-loop transfer function $\widetilde{T}_{h e c f}(s)$ is defined by substituting $Y_{h c}$ in 62 with a transfer function

$\tilde{Y}_{h c}=\tilde{Y}_{h}+\tilde{Y}_{c}$

containing the parameter uncertainties. This in turn leads to the following expression:

$\widetilde{T}_{h e c f}(s)=\frac{\left(\frac{Y_{h c}}{\widetilde{Y}_{h c}}\right) T_{h e c f}}{1+\left(\frac{Y_{h c}}{\widetilde{Y}_{h c}}-1\right) T_{h e c f}}$

Thus the perturbed system will be stable if the characteristic equation of 64 has no roots in the RHP.

We define $\delta k_{h}$ as the uncertainty in the hip joint stiffness value and $\delta k_{c}$ as the uncertainty in the coupling stiffness value. In order to study the dependency of the system's stability on $\delta k_{h}$ and $\delta k_{c}$, we will use the following intermediate expressions:

$Y_{h}=\frac{s}{D_{h}}, \quad \widetilde{Y}_{h}=\frac{s}{\widetilde{D}_{h}}, \quad Y_{c}=\frac{s}{D_{c}}, \quad \widetilde{Y}_{c}=\frac{s}{\widetilde{D}_{c}}$

where

$D_{h}=I_{h} s^{2}+b_{h} s+k_{h}, \quad \widetilde{D}_{h}=D_{h}+\delta k_{h}$

$D_{c}=b_{c} s+k_{c}, \quad \widetilde{D}_{c}=D_{c}+\delta k_{c}$

and

$\frac{Y_{h c}}{\widetilde{Y}_{h c}}=\frac{\widetilde{D}_{h} \widetilde{D}_{c}\left(D_{h}+D_{c}\right)}{D_{h} D_{c}\left(\widetilde{D}_{h}+\widetilde{D}_{c}\right)}$

Substituting 67) in 64, we arrive at the following equivalent expressions for the characteristic equation of (64):

$1+\delta k_{h} W_{h}(s)=0 \quad$ for $\delta k_{h} \neq 0, \quad \delta k_{c}=0$

$1+\delta k_{c} W_{c}(s)=0 \quad$ for $\delta k_{h}=0, \quad \delta k_{c} \neq 0$

where

$\begin{aligned} W_{h}(s) & =\frac{D_{h}+D_{c} T_{h e c f}}{D_{h}\left(D_{h}+D_{c}\right)} \\ W_{c}(s) & =\frac{D_{c}+D_{h} T_{h e c f}}{D_{c}\left(D_{h}+D_{c}\right)}\end{aligned}$

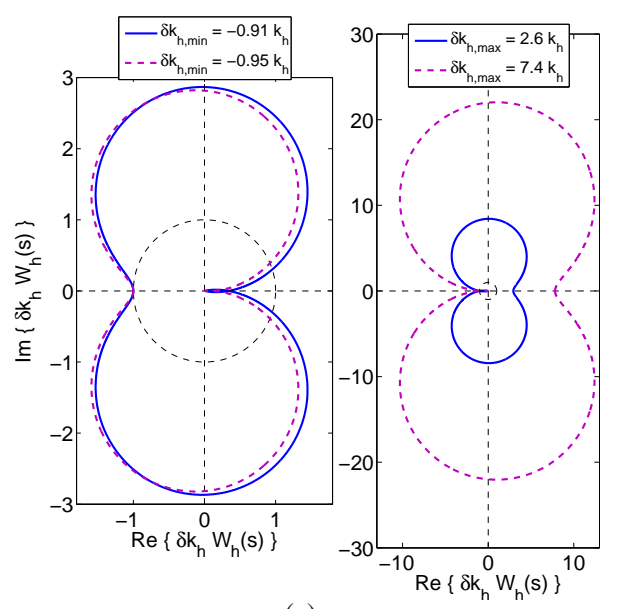

(a)

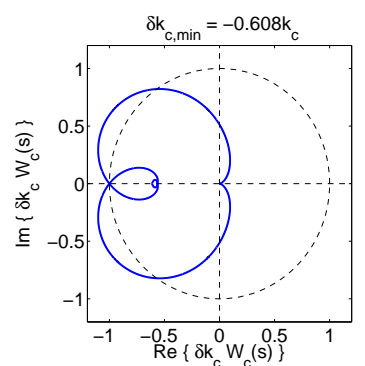

(b)

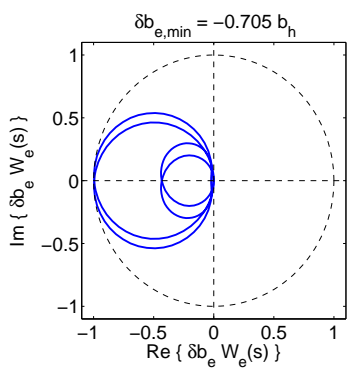

(c)
Fig. 14 Nyquist plots for the analysis of the stability robustness of the human-exoskeleton system. (a) Nyquist plots for extremal cases of hip joint stiffness variation $\delta k_{h}$. Two sets of design specifications are shown: (I) $R_{\omega}=1.2, R_{M}=1.3$ and $R_{D C}=1.1$ (continuous graphs) and (II) $R_{\omega}=1.1, R_{M}=1.2$ and $R_{D C}=1.05$ (dotted graphs). Nominal joint stiffness $\omega_{n, e c}=50$ in both cases. (b) Nyquist plot for extremal negative joint stiffness variation $\delta k_{c}$ with design specification I. (c) Nyquist plot for extremal negative exoskeleton damping variation $\delta b_{e}$ with design specification I.

\subsubsection{Hip joint stiffness}

The system's stability robustness to variations in hip joint stiffness can be determined by applying the Bode stability criterion to the open-loop transfer function $\delta k_{h} W_{h}(s)$. If we define the stable range for $\delta k_{h}$ as $\left[\delta k_{h, \min }, \delta k_{h, \max }\right]$, then $\delta k_{h, \min }$ and $\delta k_{h, \max }$ are the extremal values of $\delta k_{h}$ that satisfy

$\left|\delta k_{h} W_{h}\left(j \omega_{M}\right)\right|<1$

where $\omega_{M}=\omega \mid \angle \delta k_{h} W_{h}(j \omega)=-180^{\circ}$

Figure 14(a) shows Nyquist plots for the extremal variations of $\delta k_{h}$. In general the lowest variation margin for $\delta k_{h}$ corresponds to the extremal negative values. This indicates that, for the purposes of control design, it is safer to underestimate the nominal value of joint stiffness $k_{h}$ so that the real value involves a positive variation.

Robustness to physical variations in hip joint stiffness due to muscle activation requires special attention. Our as- 


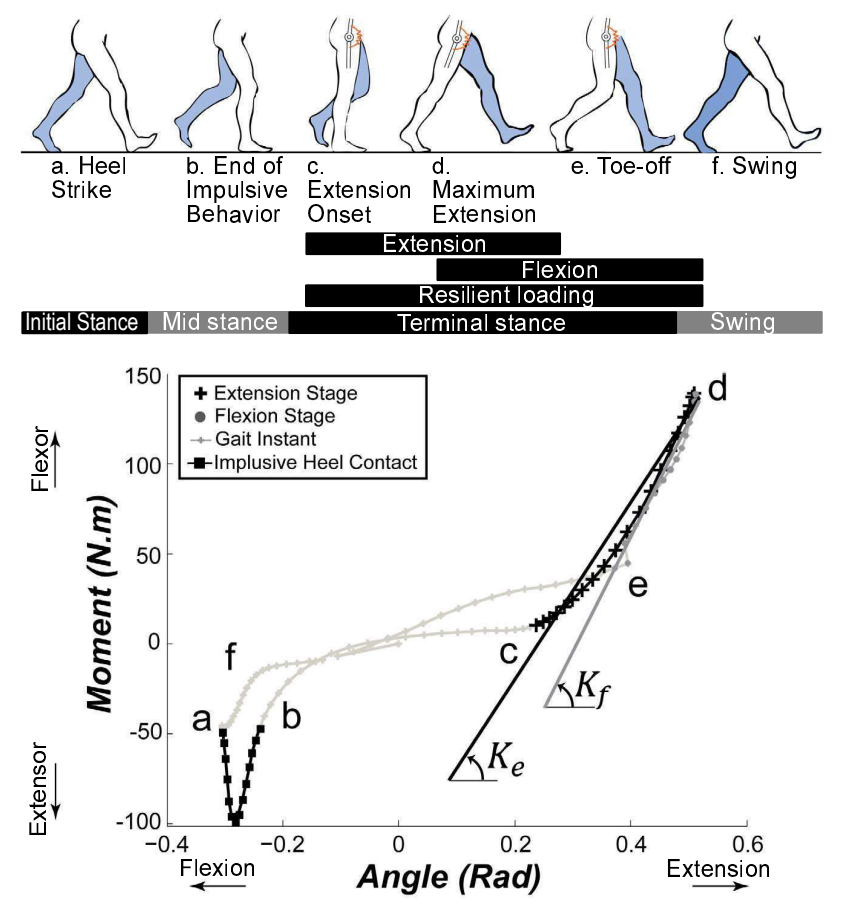

Fig. 15 Top: poses of the human leg in different phases of a walking stride. Bottom: Hip moment-angle graph for a representative human walk. Dynamic stiffness of the hip during the flexion stage (maximum extension to toe-off) is defined as the slope $K_{f}$ of the best linear fits to the portion $d-e$ of the curve. (Reproduced from Shamaei et al 2013) under the terms of the Creative Commons Attribution License.)

sistive control is optimized for the swing phase of the gait cycle; hence its parameters are based on typical hip joint stiffness values for the swing phase (Frigo et al, 1996), which are consistent with the extended leg behaving as a pendulum (Ghista, 2008; Doke et al, 2005). However, during the stance phase, the hip joint undergoes a period of loading and unloading. During this period, the hip joint's quasi-stiffness (i.e. the slope $K_{f}$ of the joint's moment-angle relationship in Figure 15) can be, on average, 9 times larger than the passive stiffness (Shamaei et al, 2013). It is thus possible that hip joint stiffness at toe-off (point $e$ in Figure 15 and early in the swing phase (portion $e-f$ ) may exceed the nominal swing-phase stiffness by a significant amount.

A large hip stiffness early in the swing phase might compromise stability and therefore limit the achievable design specifications. Figure 14(a) shows that, for the specifications of section 3.3.3 hip stiffness can only increase up to 2.6 times the nominal value before instability. By contrast, the more conservative set of design specifications in Figure 14(a) (specification II) allows for an increase 7.4 times the nominal value, which is much closer to the theoretical joint stiffness during the flexion stage of the stance phase.

We shall now consider the influence of the muscles' feedback system on the stability of the coupled system. The neuromusculoskeletal model described in Schuurmans et al (2011) considers the muscle's intrinsic impedance as well its reflex- ive feedback properties. The muscle's reflexive impedance is composed of angular velocity and angular position feedback in series with a neural time delay $\tau_{d}$ :

$$
Z_{h, r e f l}(s)=k_{p} \frac{\left(\alpha_{v} s+1\right)}{s} e^{-\tau_{d} s} \equiv k_{p} L_{h, r e f l}(s)
$$

$Z_{h, \text { refl }}$ forms a feedback loop in series with the muscle activation dynamics, given by the first-order model

$$
H_{h, a c t}(s)=\frac{1}{\tau_{a c t} s+1}
$$

In the preceding formulas, $k_{p}$ represents the muscle's reflexive stiffness and $k_{p} \alpha_{v}$ the reflexive damping; $\tau_{a c t}$ is the muscle activation time constant. To analyze coupled stability, we define a new perturbed closed-loop transfer function

$$
\widetilde{T}_{h e c f}(s)=\frac{1}{1+\left(\widetilde{Y}_{h}+Y_{c}\right) Z_{e f}}
$$

In this case $\widetilde{Y}_{h}$ represents the leg's musculoskeletal system with muscle activation feedback, and is given by

$$
\widetilde{Y}_{h}(s)=\frac{Y_{h}}{1+k_{p} H_{h, f b} Y_{h}}
$$

where $H_{h, f b}=L_{h, r e f l} H_{h, a c t}$. After substituting $\widetilde{Y}_{h}$ in (73) and performing some algebraic manipulation, we arrive at the following expression

$\widetilde{T}_{h e c f}(s)=\frac{T_{h e c f}\left(1+k_{p} H_{h, f b} Y_{h}\right)}{1+k_{p} H_{h, f b} U_{h e c f}}$

where $U_{\text {hecf }}=Y_{h}\left(1-Y_{h} Z_{\text {ef }} T_{\text {hecf }}\right)$. From (75), the robustness to reflexive stiffness is determined by the gain margin of $H_{h, f b} U_{h e c f}$. We chose a value of $0.0475 \mathrm{~s}$ for the activation time constant and $0.015 \mathrm{~s}$ for neural delay (Schuurmans et al, 2011); these constants were nondimensionalized to obtain $\tau_{a c t}$ and $\tau_{d}$. For the design specification used throughout this study (specification I in Figure 14(a), the computed gain margin is 2.95 . In other words, the reflexive stiffness could be up to 2.95 times the nominal stiffness of the hip joint, or about $110 \mathrm{Nm} / \mathrm{rad}$. Studies on balance control in humans have determined the hip reflexive stiffness to be about $95 \mathrm{Nm} / \mathrm{rad}$ (Boonstra et al, 2013). Therefore, the coupled system can in theory absorb the expected stiffening of the hip joint without loss of stability. On the other hand, for more conservative design specifications the gain margin will be even larger (for example, 8.38 for specification II.)

\subsubsection{Coupling stiffness}

Robustness to variations in coupling stiffness is determined by applying the Bode stability criterion to the open-loop transfer function $\delta k_{c} W_{c}(s)$. For positive values of $\delta k_{c}$, the phase of the Nyquist plot never reaches $180^{\circ}$ and therefore 
the variation margin for $\delta k_{c}$ is infinite, whereas for negative $\delta k_{c}$ there is the margin is finite (Figure 14(b)]. This is consistent with what we see in Figure 10, overall, to lower values of coupling stiffness $\omega_{n, e c}$ correspond lower stability margins. Therefore, like in the case of hip joint stiffness, for design purposes it is safer to underestimate the stiffness of the coupling.

\subsection{Robustness to exoskeleton damping}

Ideally, the inner-loop control should make the exoskeleton arm behave as a pure inertia (section 3.2). However, we want to consider the case in which that behavior is not produced with complete accuracy. A specific question is, if the inner-loop control generates a certain amount of negative damping instead of dead-on zero damping, will that destabilize the coupled human-exoskeleton system? For one thing, we know that, when assistance is on $\left(I_{c}>0\right)$, there is already negative damping present, as the exoskeleton's port impedance has a negative real part. Yet the coupled system remains theoretically stable. The question is then whether unintended negative damping from the inner-loop control might, in a sense, push the system over the limit and make it unstable.

To answer that question, we generate another perturbed version of $T_{\text {hecf }}$ by substituting $Z_{\text {ef }}$ in 62 with $\widetilde{Z}_{e f}=$ $Z_{e f}+\delta b_{e}$, where $\delta b_{e}$ is the variation from the ideal zero exoskeleton damping. It can be shown that the resulting transfer function $\widetilde{T}_{h e c f}$ has a characteristic equation

$1+\delta b_{e} W_{e}(s)=0$

where

$W_{e}(s)=\frac{1-T_{h e c f}}{Z_{e f}}$

Figure 14(c) shows the Nyquist plot of $\delta b_{e} W_{e}(s)$ for the extremal negative variation $\delta b_{e, \min }$. Clearly, there is a considerable stability margin for negative exoskeleton damping; the magnitude of $\delta b_{e}$ could in theory be about $70 \%$ the human damping $b_{h}$ without loss of stability. On the other hand, there is an infinite stability margin for positive values of $\delta b_{e}$. In other words, positive exoskeleton damping would only increase the stability of the coupled system, albeit at the cost of reduced performance due to additional energy dissipation.

4.3 Robustness to time delay in the exoskeleton control and other nonlinearities

Time delays in the exoskeleton control, such as are introduced by sensing and estimation, can be additional sources

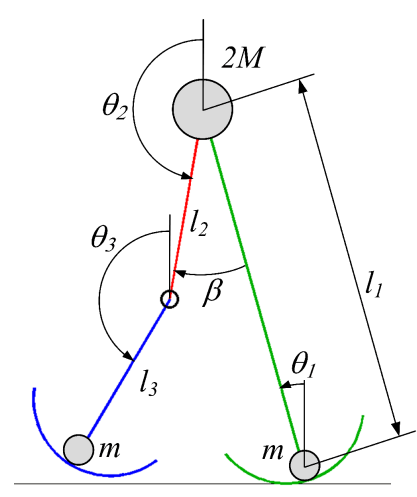

(a)

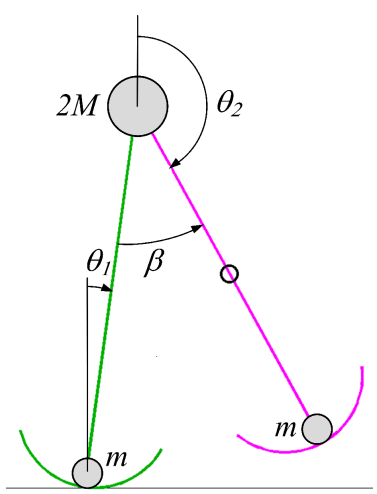

(b)
Fig. 16 Dynamic walker (DW) model. The DW's gait undergoes two phases: (a) leg swing with knee joint passively flexing and (2) leg swing with knee joint locked.

of instability. This, in turn, may require verifying the control's phase margins. For example, in the SMA device (Figure 1(a), the angular position of the motor's output shaft is measured by an array of Hall effect sensors with a resolution (gearing considered) of 600 counts per revolution. In order to eliminate quantization noise, angular velocity and acceleration of the shaft are estimated using a model-free Kalman filter (Belanger et al, 1998). For a $1.0 \mathrm{~Hz}$ sinusoidal motion, the delay in the acceleration estimate is between 4 and 5 time steps, or about $16^{\circ}$ phase.

Figure 12 (c) shows that, for the feedback compensator example in section 3.4.2, the phase margin is infinite; in other words, a pure phase delay cannot cause instability. However, for a more demanding design specification this may not be the case. In such a situation, a possible strategy can be to include a linearized model of the acceleration estimator in the feedback compensator model (50), i.e. make the estimator part of the control design.

On the other hand, nonlinearities such as backlash or actuator could give rise to limit cycles. In the SMA itself, motor torque is delivered through a custom-designed harmonic drive with a gearing ratio of 10:1. The drive as such is virtually backlash-free and therefore less likely to cause limit cycling than a conventional gear transmission. However, if limit cycles do arise they can be counteracted by the innerloop control (section 3.2), for example through the use of a lead compensator.

\section{Assisting human gait: bipedal walk simulations and initial trials with the exoskeleton}

The use of our assistive control for actual walking will be the object of a separate study. Here we offer a few initial considerations on assisting bipedal gait. We simulated the assistive control on a previously published dynamical bipedal walking model (Aguirre-Ollinger, 2014). The dynamic walker 


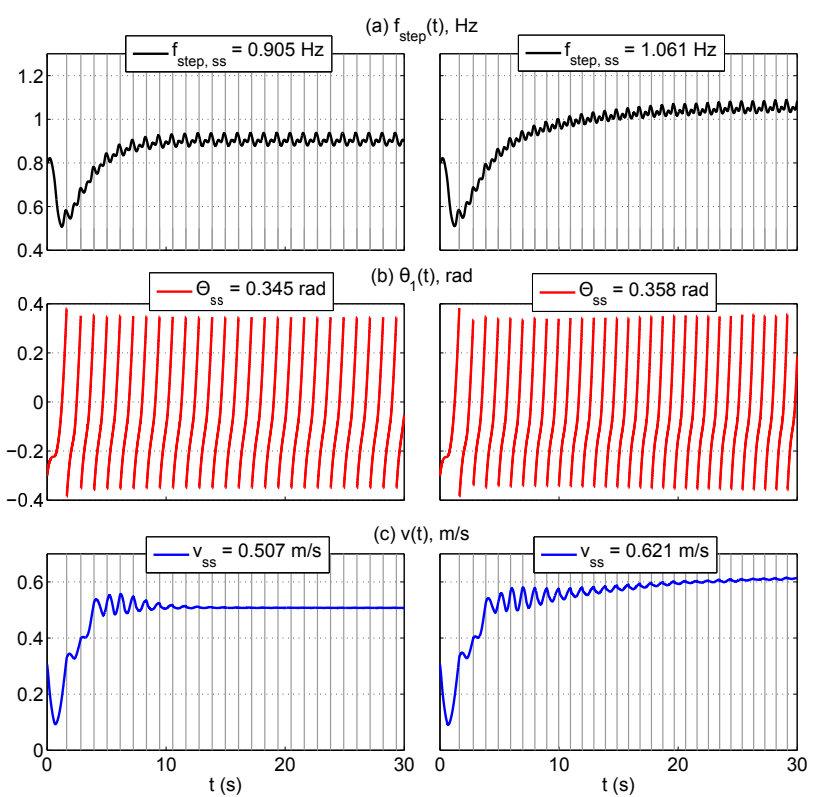

Fig. 17 Simulation results for the DW. Graphs on the left column correspond to the DW walking unassisted; graphs on the right represent the DW walking aided by a hip exoskeleton similar to the SMA device. (a) Instantaneous stepping frequency. (b) Time trajectory of the stance leg angle. (c) Horizontal speed $v(t)$ of the hip joint; $v(t)$ equals the instantaneous horizontal velocity averaged over two step cycles.
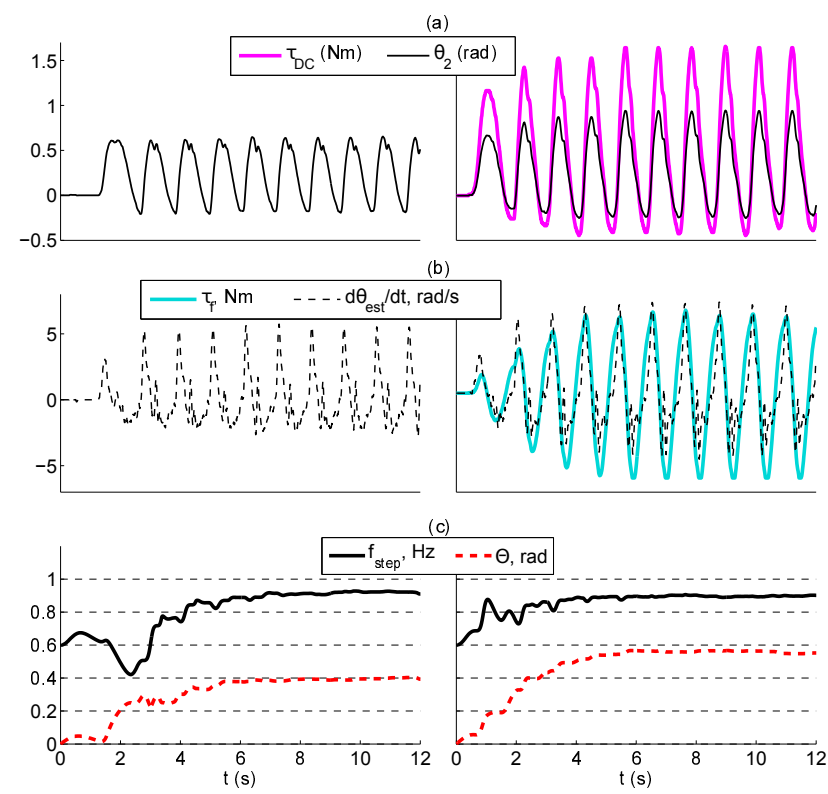

Fig. 18 Experimental trial with the SMA device: data from the right leg's motion for one subject. First row: angular trajectory $\theta_{\text {est }}(t)$ of the thigh and assistive torque $\tau_{D C}(t)$ generated by the feedback compensator for target DC gain. Second row: angular velocity $\dot{\theta}_{\text {est }}(t)$ of the thigh and assistive torque $\tau_{f}(t)$ generated by the feedback compensator for target natural frequency and resonant peak. Third row: instantaneous amplitude $\Theta(t)$ and frequency $f_{\text {step }}(t)$ of the leg's motion during walking.

(DW), shown in Figure 16, features masses concentrated at the hip and the knees as well as articulated knee joints. In lieu of hip joint muscles, the DW propels itself forward by means of bell-shaped torque profiles acting on the hips. The knee joints feature torsional springs and dampers, and bend passively during the gait cycle, thereby allowing the feet to clear the ground. The model attempts to track a given stride amplitude $\beta_{e q}$ by stiffening the hip joint after knee-lock. Appendix C provides details on the walker's kinematic and dynamic parameters as well as its mode of propulsion.

We simulated the effect of attaching a hip exoskeleton with the mass and inertia properties of the SMA device to the DW. The modeled device runs under the assistive control derived in section 3.4.1. The objective was twofold: firstly, to verify whether the model can walk stably under the action of the exoskeleton's control and, secondly, whether the effect on the gait kinematics is consistent with the predicted frequency response of the swing leg (Figure 13). This particular simulation involves no adaptation of the hip "muscle" torques to exoskeleton's action. Thus the model is expected to behave as if the exoskeleton was supplementing, rather than substituting, the hip muscles' effort.

Figure 17 shows the results of the DW simulated gaits, first unassisted, then aided by the exoskeleton. In the assisted case, the steady-state stepping frequency was $17.2 \%$ higher than in the unassisted case. This is consistent with the target natural frequency increase of $20 \%$ (section 3.3.3). From the stance length angle trajectory (Figure 17b)), variation in stride amplitude $\Theta$ between the two cases is minimal; this is expected because the model attempts to track a fixed stride amplitude. Finally, the forward speed of the model was $22.5 \%$ higher; clearly the main contribution to this increase was the change in stepping frequency.

In previous studies, human subjects performed lowerlimb swing exercises with the leg's shank coupled to a stationary 1-DOF exoskeleton (Aguirre-Ollinger, 2009; AguirreOllinger et al, 2012). The assistive control of that device was conceptually equivalent to the admittance shaping control presented here, save for the design optimization. In the baseline state, the significant inertia of the device caused a reduction in swing frequency. With the assistive function active, the inertia compensation effect enabled the subjects to recover their normal frequency and increase their selected angular velocity. The assistive action of the exoskeleton was evidenced by moderate reductions in EMG activity as well as catch trials during the experiments.

As part of the present study, we conducted a short experimental trial involving human participants using Honda's SMA device with the admitttance shaping control. Three male adult subjects walked $14 \mathrm{~m}$ on a straight-line trajectory at a uniform speed, first with the exoskeleton inactive and then with the assistive control in operation. In order to make adaptation to the SMA easy we chose conservative design specifications for the feedback compensator: $R_{\omega}=$ $1.05, R_{M}=1.05$ and $R_{D C}=1.05$. Figure 18 shows the 
experimental data for one of the participants. Subjects reported no difficulty walking with the assistive control. The main observable difference between conditions was an increase in motion amplitude, which translates into a larger stride length. This is likely a direct effect of the resonant peaks ratio $R_{M}$; the net muscle torque is supplemented by the exoskeleton torque, resulting in increased amplitude of movement.

The purpose of this experiment was only to verify the participants' ability to walk stably in the exoskeleton. Experimental validation of the kinematic and physiological effects of the assistive control on human gait requires a more elaborate experiment involving a larger number of participants walking with and without the exoskeleton for extended periods (Ferris et al, 2007).

\section{Discussion}

We have presented a method for exoskeleton assistance based on producing a virtual modification of the dynamic properties of the lower limbs. Our control formulation defines assistance as an improvement in the performance characteristics of an LTI system representing the human leg, with the desired performance defined by a sensitivity transfer function modulating the natural admittance of the leg (equation (6)).

One of the most significant aspects of our control method is that it breaks away from a performance boundary that appears often in control design literature. This boundary is stated in terms of the Bode sensitivity integral theorem by saying that, in most stable feedback control systems, the logarithmic integral of sensitivity evaluates to zero (Astrom and Murray, 2008; Stein, 2003). This property, sometimes referred to as the "waterbed effect", is seen mainly as a conservation law that puts limits to what can be achieved via feedback control. For example, servo control typically aims to reduce a system's sensitivity to disturbances. For that case the waterbed effect dictates that a reduction in sensitivity at certain frequencies is necessarily accompanied by an increase in sensitivity at other frequencies. Thus the latter is seen as an unavoidable loss of performance.

In our case the desired performance is the exact opposite: we seek to increase the system's sensitivity. In fact, the main contention of this study is that, for genuine assistance to occur, the exoskeleton control has to be capable of producing a net increase in sensitivity, i.e. we need to avoid the waterbed effect altogether. This is the case when we want to emulate effects like reduction in the inertia or the damping of the leg. 2 . Our challenge was to accomplish this with an interaction controller (as opposed to a servo controller).

\footnotetext{
2 Stiffness reduction (equivalently, increase in DC gain) stands apart because it obeys the zero sensitivity integral property.
}

Interestingly enough, it was a lesser-known part of the same integral theorem (the case for $N_{r}=1$ in (8)) that suggested a route for increasing sensitivity; namely, the use of positive feedback.

The relationship between positive feedback and assistance can also be understood in terms of work performed by the exoskeleton. The real part of the exoskeleton's port impedance is negative for frequencies in the typical range of human motion. The physical interpretation of this behavior is that the exoskeleton's port impedance possesses negative damping, causing the exoskeleton to act as an energy source rather than a dissipator. This enables the exoskeleton to perform net positive work on the leg at every stride. Thus our proposed control departs from a well-known approach to the design of robotic systems for interaction with humans; namely, that in order to guarantee the stability the robot should display passive port impedance (Colgate and Hogan, 1989b).

Although passivity is a sound principle from the point of view of safety, it is of limited usefulness for exoskeletons because a passive exoskeleton can at best provide temporary energy storage. For example, the gravity-balancing leg exoskeleton developed by Agrawal et al (2007) uses springs to make the assisted leg's potential energy invariant. However, its effect appears to be limited to increasing the leg's range of motion on impaired subjects. A passive exoskeleton could in theory reduce metabolic energy consumption during the negative work phases of muscle activation (lengthening contractions). But since muscular efficiencies are considerably lower for positive muscle work (25\%, versus $120 \%$ for negative work (Ferris et al, 2007)), a much larger economy of metabolic consumption should be expected from assisting the positive work phases instead. The MIT quasi-passive exoskeleton (Walsh et al, 2006) attempted this by storing energy during the negative-work phases of the gait cycle via springs and releasing it during the positive-work phases. However, metabolic studies showed that the device actually caused a $10 \%$ increase in metabolic cost of transport, probably as a result of the mass added by the device.

Certainly our feedback compensators achieve active behavior at the cost of some reduction in stability margins. But, as stated before, our control does not need to ensure stable interaction with every possible environment like passivity controllers do. Here stability only needs to be guaranteed for a subset of environments with impedance characteristics equal or similar to those of the human leg. For such environments we were able to find solution regions that simultaneously satisfied our performance and stability requirements (Figure 10). This led to formulating the control design as a constrained optimization problem: maximizing the gain margins of the coupled leg-exoskeleton system while enforcing the desired location of the system's dominant poles. 
A key point in our formulation is that positive feedback alone does not produce the desired performance. With pure positive feedback of the angular acceleration no region of simultaneous performance and stability exists; the system can at most cancel the exoskeleton's inertia before becoming unstable (section 3.3.3). The second-order filter in the feedback compensator $Z_{f}(s)$ (50) is absolutely necessary to generate regions of simultaneous performance and stability, i.e. regions where the dominant poles of the closed-loop system are at their target locations and the system is stable (Figure 10). The purpose of the second-order filter can be readily understood in terms of the root locus: the compensator poles $-\sigma_{f} \pm j \omega_{d, f}$ shape the system's root locus in such a way that it passes through the location of the target dominant poles ( $p_{h}^{d}$ in Figure 11). Thus the right way to think about the second-order filter in this application is as a pole placement device rather than a device for blocking frequency content.

An interesting observation is that the feedback compensator fulfills its role despite the fact that the objectives of performance and stability conflict with each other. The conflict is illustrated by Figure 11 If the inertia compensation gain $I_{c}$ is raised gradually, as one pair of poles moves towards the target locations, another pair of poles moves towards the RHP. But with proper design the target location is reached first.

A point to consider is how to validate experimentally that the assistive control achieves the target frequency response for the leg (Figure 13). The main technical challenge in this regard is how to estimate the net muscle torque, i.e. the input to $Y_{\text {hecf }}$. One possibility is to employ an experimental setup similar to the one used in Doke and Kuo (2007); in that device, muscle torque is estimated from ground reaction force measurements.

The present study dealt mostly with two control aspects: performance defined by a target frequency-response model, and stability robustness. In future work we will analyze the control's effect on transient response as well, as it may have bearing on the subject's ability to execute quick, point-topoint leg movements. We will expand the robustness analysis to include performance as well. Specifically, we want to determine how the choice of a performance target affects the controller's ability to achieve simultaneous performance and stability.

For our control formulation we modeled the human limb as a linear pendulum. Despite its simplicity, this model has proven effective in capturing the energetic properties of leg swing (Doke and Kuo, 2007). Accordingly, the scope of application of the control is assisting movements that involve muscle activation mainly at the hip joint, and no ground contact with the foot. Examples of these include the swing phase of the gait cycle, gait initiation and reactive stepping. In future studies we will conduct experiments with with human participants using an exoskeleton with the admittance shaping control. The goal of those studies will be to determine which of the possible assistive effects in our classification (section 1.1) can be achieved to a significant level by the control. The main effects we expect to see are reduction in the activation levels of the hip joint muscle groups for a given speed of walking, and subjects being able to walk comfortably at higher than normal speeds, mainly through an increase in mean stepping frequency.

Another future development is to expand our control formulation to a more realistic, multibody formulation suitable for a leg and exoskeleton with multiple degrees of freedom. Although a number of existing devices besides the SMA assist only a single ioint (Dollar and Herr, 2008a; Sawicki and Ferris, 2009), the effects on adjacent joints need to be considered as well. In the case of the SMA featuring our admittance shaping control, higher control gains might induce an oscillatory mode on the knee joint. Addressing this possibility requires, at a minimum, using a two-inertia linear model of the limb to design the control. Eventually, the admittance shaping control will have to be formulated with a proper nonlinear multibody model. Assistance in that case can be formulated as a reduction of the port impedance of the leg-exoskeleton system at the human hip joint, expressed for example as a contraction of the inertia ellipsoid.

Equally important, the control method needs to be linked to the biomechanics of human walk. In an initial study, we simulated the admittance shaping control assisting a bipedal walking model (section 5). Clearly, this model is a considerable simplification of the dynamics of human walk and omits certain aspects like ankle propulsion. However, it shows the feasibility of generating stable gait cycles with the assistive control, together with gait properties that are consistent with the target frequency response of the leg.

We also plan to extend our control formulation to assist the stance phase of walking. This is important because propulsion of the body's center of mass (CoM) takes place mainly during this phase. Kuo (2002) compared between powering a walking model via impulsive forces acting on the ankles, and using hip torques. In principle, both approaches are feasible, although hip actuation is about four times more energetically costly. However, in the case of an exoskeleton, the energetic advantage of powering the ankles may be offset by the attaching of distal masses to the legs for the purposes of ankle actuation.

Inasmuch as positive acceleration feedback makes the exoskeleton an energy source, our control approach can assist the hips during stance as well. However, since the present control is optimized for pendulum-like leg swing, a different human body model needs to be used for the design of the stance-phase control. At a minimum, the human body should be modeled as a two-body system representing the stance leg and the trunk coupled by the hip joint. 


\section{References}

Agrawal S, Banala S, Fattah A, Sangwan V, Krishnamoorthy V, Scholz J, Wei-Li H (2007) Assessment of motion of a swing leg and gait rehabilitation with a gravity balancing exoskeleton. Neural Systems and Rehabilitation Engineering, IEEE Transactions on 15(3):410-420, DOI 10.1109/TNSRE.2007.903930

Aguirre-Ollinger G (2009) Active impedance control of a lower-limb assistive exoskeleton. dissertation, Northwestern University, Evanston, IL

Aguirre-Ollinger G (2013) Learning muscle activation patterns via nonlinear oscillators: Application to lower-limb assistance. In: Intelligent Robots and Systems (IROS), 2013 IEEE/RSJ International Conference on, pp 11821189

Aguirre-Ollinger G (2014) Globally stable control of a dynamic bipedal walker using adaptive frequency oscillators. Robotica 32(7):1039-1063

Aguirre-Ollinger G (2015) Exoskeleton control for lowerextremity assistance based on adaptive frequency oscillators: Adaptation of muscle activation and movement frequency. Proceedings of the Institution of Mechanical Engineers, Part H: Journal of Engineering in Medicine 229(1):52-68, DOI 10.1177/0954411914567213

Aguirre-Ollinger G, Colgate J, Peshkin M, Goswami A (2007) A 1-DOF assistive exoskeleton with virtual negative damping: effects on the kinematic response of the lower limbs. In: IEEE/RSJ International Conference on Intelligent Robots and Systems IROS 2007, pp 1938-1944

Aguirre-Ollinger G, Colgate J, Peshkin M, Goswami A (2011) Design of an active one-degree-of-freedom lowerlimb exoskeleton with inertia compensation. The International Journal of Robotics Research 30(4):486-499

Aguirre-Ollinger G, Colgate J, Peshkin M, Goswami A (2012) Inertia compensation control of a one-degree-offreedom exoskeleton for lower-limb assistance: Initial experiments. Neural Systems and Rehabilitation Engineering, IEEE Transactions on 20(1):68-77

American Honda Motor Co, Inc (2009) Honda - walk assist and mobility devices. URL corporate.honda.com/innovation/walk-assist

Astrom KJ, Murray RM (2008) Feedback Systems: An Introduction for Scientists and Engineers. Princeton University Press, Princeton, NJ, USA

Banala S, Agrawal SK, Fattah A, Krishnamoorthy V, Hsu WL, Scholz J, Rudolph K (2006) Gravity-balancing leg orthosis and its performance evaluation. IEEE Transactions on Robotics 22(6):1228-1239

Banala S, Kim S, Agrawal S, Scholz J (2009) Robot assisted gait training with active leg exoskeleton (ALEX). Neural Systems and Rehabilitation Engineering, IEEE Transactions on 17(1):2-8
Belanger P, Dobrovolny P, Helmy A, Zhang X (1998) Estimation of angular velocity and acceleration from shaft-encoder measurements. The International Journal of Robotics Research 17(11):1225-1233

Bernhardt M, Frey M, Colombo G, Riener R (2005) Hybrid force-position control yields cooperative behaviour of the rehabilitation robot lokomat. Rehabilitation Robotics, 2005 ICORR 2005 9th International Conference on pp 536-539

Blaya J, Herr H (2004) Adaptive control of a variableimpedance ankle-foot orthosis to assist drop-foot gait. Neural Systems and Rehabilitation Engineering, IEEE Transactions on 12(1):24-31

Boonstra T, Schouten A, van der Kooij H (2013) Identification of the contribution of the ankle and hip joints to multi-segmental balance control. Journal of NeuroEngineering and Rehabilitation 10(23):1-18

Buerger S, Hogan N (2007) Complementary stability and loop shaping for improved human-robot interaction. Robotics, IEEE Transactions on 23(2):232-244

Colgate E, Hogan N (1989a) The interaction of robots with passive environments: Application to force feedback control. Fourth International Conference on Advanced Robotics

Colgate J, Hogan N (1988) Robust control of dynamically interacting systems. International Journal of Control 48(1):65-88

Colgate J, Hogan N (1989b) An analysis of contact instability in terms of passive physical equivalents. Proceedings of the IEEE International Conference on Robotics and Automation pp 404-409

Doke J, Kuo AD (2007) Energetic cost of producing cyclic muscle force, rather than work, to swing the human leg. Journal of Experimental Biology 210:2390-2398

Doke J, Donelan JM, Kuo AD (2005) Mechanics and energetics of swinging the human leg. Journal of Experimental Biology 208:439-445

Dollar A, Herr H (2008a) Design of a quasi-passive knee exoskeleton to assist running. 2008 IEEE/RSJ International Conference on Intelligent Robots and Systems pp 747-754

Dollar A, Herr H (2008b) Lower extremity exoskeletons and active orthoses: Challenges and state of the art. IEEE Transactions on Robotics 24(1):144-158

Ekso Bionics $^{\mathrm{TM}}$ (2013) Ekso bionics - an exoskeleton bionic suit or a wearable robot that helps people walk again. URL www.eksobionics.com

Emken J, Wynne J, Harkema S, Reinkensmeyer D (2006) A robotic device for manipulating human stepping. Robotics, IEEE Transactions on 22(1):185-189

European Commission (CORDIS) (2013) Balance Augmentation in Locomotion, through Anticipative, Natural and Cooperative control of Exoskeletons (BALANCE). URL 
cordis.europa.eu/projects/rcn/106854_en.html

Fee J, Miller F (2004) The leg drop pendulum test performed under general anesthesia in spastic cerebral palsy. Developmental Medicine and Child Neurology 46:273-281

Ferris D, Sawicki G, Daley M (2007) A physiologist's perspective on robotic exoskeletons for human locomotion. International Journal of Humanoid Robotics 4:507-528

Frigo C, Crenna P, Jensen L (1996) Moment-angle relationship at lower limb joints during human walking at different velocities. Journal of Electromyography and Kinesiology 6(3):177-190

Ghista D (2008) Applied Biomedical Engineering Mechanics. CRC Press

Gordon K, Kinnaird C, Ferris D (2013) Locomotor adaptation to a soleus EMG-controlled antagonistic exoskeleton. Journal of Neurophysiology 109(7):1804-1814

Hogan N, Buerger S (2006) Relaxing passivity for humanrobot interaction. Proceedings of the 2006 IEEE/RSJ International Conference on Intelligent Robots and Systems

Hogan N, Krebs HI, Rohrer B, Palazzolo J, Dipietro L, Fasoli S, Stein J, Hughes R, Frontera W, Lynch D, Volpe B (2006) Motions or muscles? some behavioral factors underlying robotic assistance of motor recovery. Journal of rehabilitation research and development 43(5):605

Kawamoto H, Sankai Y (2005) Power assist method based on phase sequence and muscle force condition for HAL. Advanced Robotics 19(7):717-734

Kawamoto H, Lee S, Kanbe S, Sankai Y (2003) Power assist method for HAL-3 using EMG-based feedback controller. In: Systems, Man and Cybernetics, 2003. IEEE International Conference on, vol 2, pp 1648-1653 vol.2

Kazerooni H, Racine J, Huang R Land Steger (2005) On the control of the berkeley lower extremity exoskeleton (BLEEX). In: Proceedings of the IEEE International Conference on Robotics and Automation ICRA 2005, pp 4353-4360

Kuo AD (2002) Energetics of actively powered locomotion using the simplest walking model. Journal of Biomechanical Engineering 124:113-120

Lee S, Sankai Y (2003) The natural frequency-based power assist control for lower body with HAL-3. IEEE International Conference on Systems, Man and Cybernetics 2:1642-1647

Lenzi T, Carrozza M, Agrawal S (2013) Powered hip exoskeletons can reduce the user's hip and ankle muscle activations during walking. Neural Systems and Rehabilitation Engineering, IEEE Transactions on 21(6):938-948

Lewis C, Ferris D (2011) Invariant hip moment pattern while walking with a robotic hip exoskeleton. Journal of Biomechanics 44(5):789-793

Malcolm P, Derave W, Galle S, De Clercq D (2013) A simple exoskeleton that assists plantarflexion can reduce the metabolic cost of human walking. PLoS ONE 8(2):e56,137, DOI 10.1371/journal.pone.0056137

Middleton R, Braslavsky J (2000) On the relationship between logarithmic sensitivity integrals and limiting optimal control problems. Decision and Control, 2000 Proceedings of the 39th IEEE Conference on 5:4990-4995 vol.5

Mooney L, Rouse E, Herr H (2014) Autonomous exoskeleton reduces metabolic cost of human walking during load carriage. Journal of NeuroEngineering and Rehabilitation 11(1):80

More J, Sorensen D (1983) Computing a trust region step. SIAM Journal on Scientific and Statistical Computing 4(3):553-572

Nef T, Mihelj M, Riener R (2007) ARMin: a robot for patient-cooperative arm therapy. Medical \& Biological Engineering \& Computing 45(9):887-900

Norris J, Granata KP, Mitros MR, Byrne EM, Marsh AP (2007) Effect of augmented plantarflexion power on preferred walking speed and economy in young and older adults. Gait \& Posture 25:620-627

Petric T, Gams A, Ijspeert A, Zlajpah L (2011) On-line frequency adaptation and movement imitation for rhythmic robotic tasks. International Journal of Robotics Research 30(14):1775-1788

Ronsse R, Lenzi T, Vitiello N, Koopman B, van Asseldonk E, De Rossi SM, van den Kieboom J, van der Kooij H, Carrozza M, Ijspeert A (2011) Oscillator-based assistance of cyclical movements: model-based and model-free approaches. Medical \& Biological Engineering \& Computing 49(10): 1173-1185

Royer TD, Martin PE (2005) Manipulations of leg mass and moment of inertia: Effects on energy cost of walking. Medicine and Science in Sports and Exercise 37(4):649656

Sawicki G, Ferris D (2008) Mechanics and energetics of level walking with powered ankle exoskeletons. Journal of Experimental Biology 211:1402-1413

Sawicki G, Ferris D (2009) Powered ankle exoskeletons reveal the metabolic cost of plantar flexor mechanical work during walking with longer steps at constant step frequency. Journal of Experimental Biology 212:21-31

Schouten A, Vlugt E, Van der Helm F (2008) Design of perturbation signals for the estimation of proprioceptive reflexes. IEEE Transactions on Biomedical Engineering 55(5):1612-1619

Schuurmans J, van der Helm F, Schouten A (2011) Relating reflex gain modulation in posture control to underlying neural network properties using a neuromusculoskeletal model. Journal of Computational Neuroscience 30(3):555-565

Shamaei K, Sawicki G, Dollar A (2013) Estimation of quasistiffness of the human hip in the stance phase of walking. PLoS ONE 8(12):e81,841, DOI 10.1371/journal.pone. 


\section{1}

Stein G (2003) Respect the unstable. Control Systems, IEEE 23(4):12-25

Sugar T, Bates A, Holgate M, Kerestes J, Mignolet M, New P, Ramachandran R, Redkar S, Wheeler C (2015) Limit cycles to enhance human performance based on phase oscillators. Journal of Mechanisms and Robotics 7(1):8 pages, DOI 10.1115/1.4029336

Tafazzoli F, Lamontagne M (1996) Mechanical behaviour of hamstring muscles in low-back pain patients and control subjects. Clinical Biomechanics 11(1):16-24

Vallery H, Duschau-Wicke A, Riener R (2009a) Generalized elasticities improve patient-cooperative control of rehabilitation robots. In: IEEE International Conference on Rehabilitation Robotics ICORR 2009, June 23-26, Kyoto, Japan, pp 535-541

Vallery H, Van Asseldonk EHF, Buss M, van der Kooij H (2009b) Reference trajectory generation for rehabilitation robots: Complementary limb motion estimation. Neural Systems and Rehabilitation Engineering, IEEE Transactions on 17(1):23-30

Van Asseldonk E, Ekkelenkamp R, Veneman J, Van der Helm F, Van der Kooij H (2007) Selective control of a subtask of walking in a robotic gait trainer(LOPES). Proceedings of the IEEE International Conference on Rehabilitation Robotics pp 841-848

Veneman J, Ekkelenkamp R, Kruidhof R, Van der Helm F, Van der Kooij H (2005) Design of a series elasticand Bowden cable-based actuation system for use as torque-actuator in exoskeleton-type training. Proceedings of the IEEE International Conference on Rehabilitation Robotics pp 496-499

Walsh C, Paluska D, Pasch K, Grand W, Valiente A, Herr H (2006) Development of a lightweight, underactuated exoskeleton for load-carrying augmentation. In: Proceedings IEEE International Conference on Robotics and Automation ICRA 2006, May 15-19, Orlando, FL, USA, pp 3485 $-3491$

\section{A Target values for the dynamic response parameters of the assisted leg: computation}

From 14 we have

$\omega_{n h}^{d}=R_{\omega} \omega_{n h}$

We shall now define an intermediate target integral admittance $X_{h, D C}(s)$ that differs from $X_{h}(s)$ only in the trailing coefficient of the denominator:

$X_{h, D C}(s)=\frac{1}{I_{h} s^{2}+2 I_{h} \zeta_{h} \omega_{n h} s+I_{h} \omega_{n h, D C}^{2}}$
We choose $\omega_{n h, D C}$ such that $X_{h, D C}(s)$ meets the DC gain specification $R_{D C}$ :

$\frac{X_{h, D C}(0)}{X_{h}(0)}=\frac{\omega_{n h}^{2}}{\omega_{n h, D C}^{2}}=R_{D C}$

yielding

$\omega_{n h, D C}=\omega_{n h} \sqrt{R_{D C}^{-1}}$

Because the target integral admittance $X_{h}^{d}(s)$ and the intermediate target $X_{h, D C}(s)$ have the same DC gains (although in general they have different natural frequencies and different damping ratios), we can write

$X_{h}^{d}(0)=X_{h, D C}(0)$ or

$\frac{1}{I_{h}^{d} \omega_{n h}^{d 2}}=\frac{1}{I_{h} \omega_{n h, D C}^{2}}$

In 82 we substitute $\omega_{n h}^{d}$ with 78 and $\omega_{n h, D C}$ with 81, thereby obtaining the required value for $I_{h}^{d}$ :

$I_{h}^{d}=\frac{I_{h}}{R_{D C} R_{\omega}^{2}}$

In order to obtain $\zeta_{h}^{d}$, we compute the values of the resonant peaks for $X_{h}(j \omega)$ using equation 12 and $X_{h}^{d}(j \omega)$ using equation (13):

$M_{h}=\frac{1}{2 I_{h} \omega_{n h}^{2} \zeta_{h} \sqrt{1-\zeta_{h}^{2}}}$ for $X_{h}(j \omega)$

and

$M_{h}^{d}=\frac{1}{2 I_{h}^{d} \omega_{n h}^{d 2} \zeta_{h}^{d} \sqrt{1-\zeta_{h}^{d 2}}}$ for $X_{h}^{d}(j \omega)$

Computing the ratio $M_{h}^{d} / M_{h}$ and applying 83 yields

$\frac{M_{h}^{d}}{M_{h}}=\frac{R_{D C} \zeta_{h} \sqrt{1-\zeta_{h}^{2}}}{\zeta_{h}^{d} \sqrt{1-\zeta_{h}^{d 2}}}$

Equating the left-hand side of 86 to $R_{M}$ (definition 15) yields

$\zeta_{h}^{d} \sqrt{1-\zeta_{h}^{d} 2}=\frac{R_{D C}}{R_{M}} \zeta_{h} \sqrt{1-\zeta_{h}^{2}}$

Now we define the right-hand side of 87 as

$\rho=\frac{R_{D C}}{R_{M}} \zeta_{h} \sqrt{1-\zeta_{h}^{2}}$

yielding

$\zeta_{h}^{d}{ }^{4}-\zeta_{h}^{d}{ }^{2}+\rho^{2}=0$

for which the solution that ensures the existence of a resonant peak is

$\zeta_{h}^{d}=\sqrt{\frac{1-\sqrt{1-4 \rho^{2}}}{2}}$ 


\section{B Pole placement solution for the feedback compensator based on the properties of the positive-feedback root locus}

Given a target dominant pole $p_{h}^{d}$, the phase of $L_{h e c f}\left(p_{h}^{d}\right)$ is computed as

$\Phi\left(\sigma_{f}, \omega_{d, f}, p_{h}^{d}\right)=\sum_{i=1}^{N_{z}} \psi_{i}-\sum_{i=1}^{N_{p}} \phi_{i}-\phi_{f}-\bar{\phi}_{f}$

where

$$
\begin{aligned}
& \psi_{i}=\arctan \left(\frac{\operatorname{Im}\left\{p_{h}^{d}-z_{h e c, i}\right\}}{\operatorname{Re}\left\{p_{h}^{d}-z_{h e c, i}\right\}}\right) \\
& \phi_{i}=\arctan \left(\frac{\operatorname{Im}\left\{p_{h}^{d}-p_{h e c, i}\right\}}{\operatorname{Re}\left\{p_{h}^{d}-p_{h e c, i}\right\}}\right) \\
& \phi_{f}=\arctan \left(\frac{\operatorname{Im}\left\{p_{h}^{d}\right\}-\omega_{d, f}}{\operatorname{Re}\left\{p_{h}^{d}\right\}+\sigma_{f}}\right) \\
& \bar{\phi}_{f}=\arctan \left(\frac{\operatorname{Im}\left\{p_{h}^{d}\right\}+\omega_{d, f}}{\operatorname{Re}\left\{p_{h}^{d}\right\}+\sigma_{f}}\right)
\end{aligned}
$$

Here $z_{h e c, i}$ are the zeros of $L_{h e c f}(s)$ and $p_{h e c, i}$ are the poles of $L_{h e c f}(s)$ excepting those at $s=-\sigma_{f} \pm j \omega_{d, f}$. Thus $N_{p}=N_{z}=$ 4. A valid solution for $\sigma_{f}$ and $\omega_{d, f}$ satisfies $\Phi\left(\sigma_{f}, \omega_{d, f}, p_{h}^{d}\right)=0$ for positive feedback.

Given a solution for $\sigma_{f}$ and $\omega_{d, f}$, the loop gain 54 is computed as

$K_{L}\left(\sigma_{f}, \omega_{d, f}, p_{h}^{d}\right)=-\mathcal{K}_{L, f} \overline{\mathcal{K}}_{L, f} \mathcal{K}_{L, h e c}$

where

$$
\begin{aligned}
\mathcal{K}_{L, f}= & {\left[\left(\operatorname{Re}\left\{p_{h}^{d}\right\}+\sigma_{f}\right)^{2}+\left(\operatorname{Im}\left\{p_{h}^{d}\right\}-\omega_{d, f}\right)^{2}\right]^{\frac{1}{2}} } \\
\overline{\mathcal{K}}_{L, f}= & {\left[\left(\operatorname{Re}\left\{p_{h}^{d}\right\}+\sigma_{f}\right)^{2}+\left(\operatorname{Im}\left\{p_{h}^{d}\right\}+\omega_{d, f}\right)^{2}\right]^{\frac{1}{2}} } \\
\mathcal{K}_{L, h e c}= & \frac{\prod_{i=1}^{N_{p}}\left[\operatorname{Re}\left\{p_{h}^{d}-p_{h e c, i}\right\}^{2}+\operatorname{Im}\left\{p_{h}^{d}-p_{h e c, i}\right\}^{2}\right]^{\frac{1}{2}}}{\prod_{i=1}^{N_{z}}\left[\operatorname{Re}\left\{p_{h}^{d}-z_{h e c, i}\right\}^{2}+\operatorname{Im}\left\{p_{h}^{d}-z_{h e c, i}\right\}^{2}\right]^{\frac{1}{2}}}
\end{aligned}
$$

\section{Target values for the dynamic response parameters of the assisted leg: computation}

In the dynamic walker (DW) of Figure 16 described in Aguirre-Ollinger (2014), a combination of adaptive frequency oscillator (AFO) and adaptive Fourier analysis (Petric et al, 2011) generates a cyclic phase signal $\varphi(t)$. We use $\varphi$ to generate bell-shaped torques on the hip joints in lieu of hip muscle torques. The hip torque profiles for the stance and the swing phases are given, respectively, by

$$
\begin{aligned}
\tau_{s t}(\varphi) & =\frac{\tau_{s t, \text { max }}}{K_{s t}} \varphi \exp \left(1-\frac{\varphi}{K_{s t}}\right) \\
\tau_{s w}(\varphi) & =\frac{\tau_{s w, \max }}{K_{s w}} \varphi \exp \left(1-\frac{\varphi}{K_{s w}}\right)
\end{aligned}
$$

The knee joint possesses torsional stiffness $\kappa_{k n}$ and damping $\nu_{k n}$. The knee becomes locked when the leading leg reaches full extension and is released again at toe-off. In order to ensure a stable gait, the hip joint of the leading leg is stiffened during the interval from kneelock to ground collision. The torsional stiffness and damping for this interval are, respectively, $\kappa_{s w}$ and $\nu_{s w}$. The virtual spring $\kappa_{s w}$ has an equilibrium defined by an inter-leg angle $\beta=\beta_{\text {eq }}$ (Figure 16b)).

The numerical parameters employed in the simulation of section 5 are given below.

- Multibody system: $M=19.62 \mathrm{~kg}, m=15.38 \mathrm{~kg}, l_{2}=0.4165$ $\mathrm{m}, l_{3}=0.4845 \mathrm{~m}$, foot radius $=0.1 \mathrm{~m}$

- Hip joint damping: $b_{h}=2.194 \mathrm{Nms} / \mathrm{rad}$

- Stance-phase hip torque: $\tau_{s t, \max }=39.7 \mathrm{Nm}, K_{s t}=0.5$

- Swing-phase hip torque: $\tau_{s w, \max }=35.3 \mathrm{Nm}, K_{s w}=0.5$

- Hip joint stiffness and damping at the end of the swing phase $\kappa_{s w}=96 \mathrm{Nm} / \mathrm{rad}, \nu_{s w}=32 \mathrm{Nms} / \mathrm{rad}$; equilibrium point: $\beta_{\text {eq }}=$ $0.6 \mathrm{rad}$

- Knee joint stiffness and damping: $\kappa_{k n}=8.43 \mathrm{Nm} / \mathrm{rad}, \nu_{k n}=$ $0.966 \mathrm{Nms} / \mathrm{rad}$ 